\title{
On a theorem of Chernoff on rank one Riemannian symmetric spaces
}

\author{
Pritam Ganguly $^{\mathrm{a}, *}$, Ramesh Manna ${ }^{\mathrm{b}}$, Sundaram Thangavelu ${ }^{\mathrm{a}}$ \\ a Department of Mathematics, Indian Institute of Science, 560012 Bangalore, \\ India \\ b School of Mathematical Sciences, National Institute of Science Education and \\ Research Bhubaneswar, HBNI, Jatni 752050, India
}

\section{A R T I C L E I N F O}

\section{Article history:}

Received 17 June 2021

Accepted 4 December 2021

Available online 14 December 2021

Communicated by Camil Muscalu

\section{$M S C$ :}

primary 43A $85,43 \mathrm{~A} 25$

secondary 22E30, 33C45

Keywords:

Chernoff's theorem

Riemannian symmetric spaces

Helgason Fourier transform

Jacobi analysis
A B S T R A C $\mathrm{T}$

In 1975, P.R. Chernoff used iterates of the Laplacian on $\mathbb{R}^{n}$ to prove an $L^{2}$ version of the Denjoy-Carleman theorem which provides a sufficient condition for a smooth function on $\mathbb{R}^{n}$ to be quasi-analytic. In this paper we prove an exact analogue of Chernoff's theorem for all rank one Riemannian symmetric spaces of noncompact type using iterates of the associated Laplace-Beltrami operators. Moreover, we also prove an analogue of Chernoff's theorem for the sphere which is a rank one compact symmetric space.

() 2021 Elsevier Inc. All rights reserved.

\section{Introduction and the main results}

The paramount property of an analytic function is that it is completely determined by its value and the values of all its derivatives at a single point. Borel first perceived that there is a larger class of smooth functions than that of analytic functions which has

\footnotetext{
* Corresponding author.

E-mail addresses: pritamg@iisc.ac.in (P. Ganguly), rameshmanna@niser.ac.in (R. Manna), veluma@iisc.ac.in (S. Thangavelu).
} 
this magnificent property. He coined the term quasi-analytic for such class of functions. In exact terms a subset of smooth functions on an interval $(a, b)$ is called a quasi-analytic class if for any function $f$ from that set and $x_{0} \in(a, b), \frac{d^{n}}{d x^{n}} f\left(x_{0}\right)=0$ for all $n \in \mathbb{N}$ implies $f=0$. Now recall that a smooth function on an interval $I$ is analytic provided its Taylor series converges to the function on $I$ which naturally restricts the growth of derivatives of that function. In fact, if for every $n,\left\|\frac{d^{n}}{d x^{n}} f\right\|_{L^{\infty}(I)} \leq C n ! A^{n}$ for some constant $A$ depending on $f$ then the Taylor series of $f$ converges to $f$ uniformly and the converse is also true. This drives an analytic mind to investigate whether relaxing growth condition on the derivatives generates quasi-analytic class. In 1912 Hadamard proposed the problem of finding sequence $\left\{M_{n}\right\}_{n}$ of positive numbers such that the class $C\left\{M_{n}\right\}$ of smooth functions on $I$ satisfying $\left\|\frac{d^{n}}{d x^{n}} f\right\|_{L^{\infty}(I)} \leq A_{f}^{n} M_{n}$ for all $f \in C\left\{M_{n}\right\}$ is a quasi-analytic class. A solution to this problem is provided by a theorem of Denjoy and Carleman where they showed that $C\left\{M_{n}\right\}$ is quasi-analytic if and only if $\sum_{n=1}^{\infty} M_{n}^{-1 / n}=$ $\infty$. As a matter of fact Denjoy [10] first proved a sufficient condition and later Carleman [6] completed the theorem giving a necessary and sufficient condition. A short proof of this theorem based on complex analytic ideas can be found in Rudin [26]. A several variable analogue of this theorem has been obtained by Bochner and Taylor [4] in 1939.

Later in 1950, instead of using all partial derivatives, Bochner used iterates of the Laplacian $\Delta$ and proved an analogue of Denjoy-Carleman theorem which reads as follows: if $f \in C^{\infty}\left(\mathbb{R}^{n}\right)$ satisfies $\sum_{m=1}^{\infty}\left\|\Delta^{m} f\right\|_{\infty}^{-1 / m}=\infty$, then the condition $\Delta^{m} f(x)=0$ for all $m \geq 0$ and for all $x$ in a set $U$ of analytic determination implies $f=0$. Building upon the works of Masson- McClary [24] and Nussbaum [25], in 1972 Chernoff [7] used operator theoretic arguments to study quasi-analytic vectors. As an application he improved the above mentioned result of Bochner by proving the following very interesting result in 1975 .

Theorem 1.1. [8, Chernoff] Let $f$ be a smooth function on $\mathbb{R}^{n}$. Assume that $\Delta^{m} f \in$ $L^{2}\left(\mathbb{R}^{n}\right)$ for all $m \in \mathbb{N}$ and $\sum_{m=1}^{\infty}\left\|\Delta^{m} f\right\|_{2}^{-\frac{1}{2 m}}=\infty$. If $f$ and all its partial derivatives vanish at a point $a \in \mathbb{R}^{n}$, then $f$ is identically zero.

As Chernoff's theorem is a useful tool in establishing uncertainty principles of Ingham's type ([14]), proving analogues of Theorem 1.1 in contexts other than Euclidean spaces have received considerable attention in recent years. Recently, an analogue of Chernoff's theorem for the sublaplacian on the Heisenberg group has been proved in [1]. See also [13] for an analogue of Chernoff's theorem for the full Laplacian on the Heisenberg group. In this paper we prove an analogue of Chernoff's theorem for the Laplace-Beltrami operator on rank one symmetric spaces of both compact and noncompact types.

In order to state our results we first need to introduce some notations. Let $G$ be a connected, noncompact semisimple Lie group with finite centre and $K$ a maximal compact subgroup of $G$. Let $X=G / K$ be the associated symmetric space which is assumed to have rank one. The origin $o$ in the symmetric space is given by the identity coset 
$e K$ where $e$ is the identity element in $G$. We know that $X$ is a Riemannian manifold equipped with a $G$ invariant metric on it. We denote by $\Delta_{X}$ the Laplace-Beltrami operator associated to $X$. For noncompact Riemannian symmetric spaces $X=G / K$, without any restriction on the rank, the following weaker version of Theorem 1.1 has been proved in Bhowmik-Pusti-Ray [2].

Theorem 1.2 (Bhowmik-Pusti-Ray). Let $X=G / K$ be a noncompact Riemannian symmetric space and let $\Delta_{X}$ be the associated Laplace-Beltrami operator. Suppose $f \in$ $C^{\infty}(X)$ satisfies $\Delta_{X}^{m} f \in L^{2}(X)$ for all $m \geq 0$ and $\sum_{m=1}^{\infty}\left\|\Delta_{X}^{m} f\right\|_{2}^{-\frac{1}{2 m}}=\infty$. If $f$ vanishes on a non empty open set, then $f$ is identically zero.

In proving the above theorem, the authors have made use of a result of de Jeu [15]. In the case of rank one symmetric spaces, a different proof was given by the first and third authors of this article by making use of spherical means and an analogue of Chernoff's theorem for the Jacobi transform proved in [12]. In fact, we only need to use the one dimensional version of de Jeu's theorem which is equivalent to the Denjoy-Carleman theorem. Our proof of Theorem 1.7 proved in this paper is built upon the ideas used in $[12]$.

In a very recent preprint [3], Bhowmik-Pust-Ray have proved the following improvement of their Theorem 1.2. In what follows let $D(G / K)$ denote the algebra of differential operators on $G / K$ which are invariant under the (left) action of $G$.

Theorem 1.3 (Bhowmik-Pusti-Ray). Let $X=G / K$ be a noncompact Riemannian symmetric space and let $\Delta_{X}$ be the associated Laplace-Beltrami operator. Suppose $f \in$ $C^{\infty}(G / K)$ be a left $K$-invariant function on $X$ which satisfies $\Delta_{X}^{m} f \in L^{2}(X)$ for all $m \geq 0$ and $\sum_{m=1}^{\infty}\left\|\Delta_{X}^{m} f\right\|_{2}^{-\frac{1}{2 m}}=\infty$. If there is an $x_{0} \in X$ such that $\operatorname{Df}\left(x_{0}\right)$ vanishes for all $D \in D(G / K)$ then $f$ is identically zero.

Remark 1.4. Observe that in the above theorem the function $f$ is assumed to be $K$ biinvariant. It is well-known that, for rank one symmetric spaces of noncompact type, $D(G / K)$ consists of only polynomials in the Laplace-Beltrami operator which is somewhat analogous to the Euclidean case. In fact, $D(G / K)$ is the counterpart of the set of all translation and rotation invariant differential operators on $\mathbb{R}^{n}$ which are given by polynomials in Laplacian on $\mathbb{R}^{n}$. In both the cases, it has been shown that Chernoff's theorem does not hold with vanishing condition assumed only on the powers of Laplacian. Indeed, for explicit examples see [8, Page-645] for the Euclidean space and see [2, Example 3.7] for the rank one symmetric spaces. As a matter of fact, it is natural to expect that, for the right analogue of Chernoff's theorem, one needs to consider more derivatives. 
In order to motivate what we do in the case of rank one symmetric spaces, let us revisit the Euclidean case. For functions on $\mathbb{R}^{n}$ consider the condition $\left.\left(\frac{d}{d r}\right)^{k} f(r \omega)\right|_{r=0}=0$ where $x=r \omega, r>0, \omega \in \mathbb{S}^{n-1}$ is the polar coordinates of $x \in \mathbb{R}^{n}$. As can be easily checked

$$
\left(\frac{d}{d r}\right)^{k} f(r \omega)=\sum_{|\alpha|=k} \partial^{\alpha} f(r \omega) \omega^{\alpha}
$$

and hence $\left.\left(\frac{d}{d r}\right)^{k} f(r \omega)\right|_{r=0}=0$ for all $k$ if and only if $\partial^{\alpha} f(0)=0$ for all $\alpha$. This observation plays an important role in formulating the right analogue Chernoff's theorem for compact Riemannian symmetric spaces. In view of the above observation, Chernoff's theorem for the Laplacian on $\mathbb{R}^{n}$ can be stated in the following form.

Theorem 1.5. Let $f$ be a smooth function on $\mathbb{R}^{n}$. Assume that $\Delta^{m} f \in L^{2}\left(\mathbb{R}^{n}\right)$ for all $m \in \mathbb{N}$ and $\sum_{m=1}^{\infty}\left\|\Delta^{m} f\right\|_{2}^{-\frac{1}{2 m}}=\infty$. If $\left.\left(\frac{d}{d r}\right)^{k} f(r \omega)\right|_{r=0}=0$ for all $k$ and $\omega \in \mathbb{S}^{n-1}$, then $f$ is identically zero.

We can give a proof of the above theorem by reducing it to a theorem for Bessel operators. Recall that written in polar coordinates the Laplacian takes the form

$$
\Delta=\frac{\partial^{2}}{\partial r^{2}}+\frac{n-1}{r} \frac{\partial}{\partial r}+\frac{1}{r^{2}} \Delta_{\mathbb{S}^{n-1}}
$$

where $\Delta_{\mathbb{S}^{n-1}}$ is the spherical Laplacian on the unit sphere $\mathbb{S}^{n-1}$. By expanding the function $F(r, \omega)=f(r \omega)$ in terms of spherical harmonics on $\mathbb{S}^{n-1}$ and making use of Hecke-Bochner formula, we can easily reduce Theorem 1.5 to a sequence of theorems for the Bessel operator $\partial_{r}^{2}+(n+2 m+1) r^{-1} \partial_{r}$ for various values of $m \in \mathbb{N}$. This idea has been already used in the paper [12]. A similar expansion in the case of noncompact Riemannian symmetric spaces leads to Jacobi operators as done in [12] which will be used in proving Theorem 1.7. As the proof of the above theorem is similar to and easier than that of Theorem 1.7, we will not present it here.

Remark 1.6. We remark in passing that the above theorem can also be proved in the context of Dunkl Laplacian on $\mathbb{R}^{n}$ associated to root systems. We would also like to mention that analogues of Chernoff's theorem can be proved for the Hermite operator $H$ on $\mathbb{R}^{n}$ and the special Hermite operator $L$ on $\mathbb{C}^{n}$. Again the idea is to make use of Hecke-Bochner formula for the Hermite and special Hermite projections (associated to their spectral decompositions).

In the case of rank one symmetric spaces we look for the counterpart of $\left.\left(\frac{d}{d r}\right)^{k} f(r \omega)\right|_{r=0}=0$. The Iwasawa decomposition of $G$ reads as $G=K A N$ where $A$ is abelian and $N$ is a nilpotent Lie group. Let $\mathfrak{g}$ and $\mathfrak{a}$ stand for the Lie algebras corresponding to $G$ and $A$ respectively. Here $\mathfrak{a}$ is one dimensional since $X$ is of rank one. It is well known that every element of $\mathfrak{g}$ gives rise to a left invariant vector field on $G$. Let 
$H$ be the left invariant vector field corresponding to a fixed basis element of $\mathfrak{a}$. We will describe all these notations in detail in the next section.

We consider the condition $H^{l}(e K)=0$ as the analogue of $\left.\left(\frac{d}{d r}\right)^{k} f(r \omega)\right|_{r=0}=0$. To justify the analogy, we remark that this condition is equivalent to the condition $V^{l}(e K)=$ 0 for all $V \in \mathfrak{p}$ where $\mathfrak{p}$ is the tangent space of $X=G / K$ at the identity coset. This is clear from the facts that $\mathfrak{p}=\cup_{k \in K} \operatorname{Ad}_{k} \mathfrak{a}$ and

$$
H^{l} f(k)=\frac{d^{l}}{d t^{l}} f(k \exp (t H))=\frac{d^{l}}{d t^{l}} f\left(k \exp (t H) k^{-1}\right)=\frac{d^{l}}{d t^{l}} f\left(\exp \left(\operatorname{Ad}_{k} H\right)\right)
$$

for any $k \in K$. Note that none of these vector fields belong to $D(G / K)$. It would be interesting to see whether analogous vector fields can be used in the vanishing condition in the higher rank case to obtain a correct analogue of Chernoff's theorem without any extra assumptions on the function side. As an analogue of the version of Chernoff's theorem stated in Theorem 1.5 for rank one symmetric spaces $X$ we offer the following:

Theorem 1.7. Let $X=G / K$ be a rank one symmetric space of noncompact type. Suppose $f \in C^{\infty}(X)$ satisfies $\Delta_{X}^{m} f \in L^{2}(X)$ for all $m \geq 0$ and $\sum_{m=1}^{\infty}\left\|\Delta_{X}^{m} f\right\|_{2}^{-\frac{1}{2 m}}=\infty$. If $H^{l} f(e K)=0$ for all $l \geq 0$ then $f$ is identically zero.

Now in view of the translation invariance of Laplace-Beltrami operator $\Delta_{X}$ and $H$, it is not difficult to see that the above theorem holds true if we take any $x_{0} \in X$ in place of $e K$. As an immediate consequence of the above result we obtain an analogue of the $L^{2}$ version of the classical Denjoy-Carleman theorem using iterates of the Laplace-Beltrami operator on $X=G / K$.

Corollary 1.8. Let $X=G / K$ be a rank one symmetric space of noncompact type. Let $\left\{M_{k}\right\}_{k}$ be a log convex sequence. Define $\mathcal{C}\left(\left\{M_{k}\right\}_{k}, \Delta_{X}, X\right)$ to be the class of all smooth functions $f$ on $X$ satisfying $\Delta_{X}^{m} f \in L^{2}(X)$ for all $m \in \mathbb{N}$ and $\left\|\Delta_{X}^{k} f\right\|_{2} \leq M_{k} \lambda(f)^{k}$ for some constant $\lambda(f)$ depending on $f$. Suppose that $\sum_{k=1}^{\infty} M_{k}^{-\frac{1}{2 k}}=\infty$. Then every member of that class is quasi-analytic.

So far we have only considered non compact Riemannian symmetric spaces, but now we turn our attention to proving an analogue of Theorem 1.5 for compact, rank one symmetric spaces. As we will see, our investigation is complete only for the case of spheres.

Let $(U, K)$ be a compact symmetric pair and $S=U / K$ be the associated symmetric space. Here $U$ is a compact semisimple Lie group and $K$ is a connected subgroup of $U$. We assume that $S$ has rank one. Being a compact Riemannian manifold, $S$ admits a Laplace-Beltrami operator $\Delta_{S}$. In [28], H.C. Wang has completely classified all rank one compact symmetric spaces. To be more precise, $S$ is one of the followings: The unit 
sphere $\mathbb{S}^{q}=S O(q+1) / S O(q)$, the real projective space $P_{q}(\mathbb{R})=S O(q+1) / O(q)$, the complex projective space $P_{l}(\mathbb{C})$, the quaternion projective space $P_{l}(\mathbb{H})$ and the Cayley projective space $P_{2}(\mathbb{C} a y)=F_{4} / \operatorname{Spin}(9)$. In each case, $S$ comes up with an appropriate polar form $(0, \pi) \times \mathbb{S}^{q}$ where $q=q_{S}$ depends on the symmetric space $S$. As a consequence, functions on $S$ can be identified with functions on the product space $Y=(0, \pi) \times \mathbb{S}^{q}$.

In order to motivate the formulation of our result for spheres, and in general for compact rank one symmetric spaces, let us return to the Euclidean case. In the context of Theorem 1.5 , by identifying $\mathbb{R}^{n}$ with $(0, \infty) \times \mathbb{S}^{n-1}$, every function $f$ on $\mathbb{R}^{n}$ gives rise to a function $F(r, \omega)$ on $(0, \infty) \times \mathbb{S}^{n-1}$ and in view of $(1.1)$, the action of $\Delta$ on $f$ takes the form,

$$
\Delta f(r, \omega)=\frac{\partial^{2}}{\partial r^{2}} F(r, \omega)+\frac{n-1}{r} \frac{\partial}{\partial r} F(r, \omega)+\frac{1}{r^{2}} \Delta_{\mathbb{S}^{n-1}} F(r, \omega) .
$$

There is a similar decomposition of $\Delta_{\mathbb{S} q}$ as a sum of a Jacobi operator on $(0, \pi)$ and the spherical Laplacian $\Delta_{\mathbb{S} q-1}$ and this justifies our formulation of Chernoff's theorem for spheres. In order to describe this we work with the geodesic polar coordinate system on $\mathbb{S}^{q}$. Note that given $\xi \in \mathbb{S}^{q}$, we can write $\xi=(\cos \theta) e_{1}+\xi_{1}^{\prime}(\sin \theta) e_{2}+\ldots+\xi_{q}^{\prime}(\sin \theta) e_{q+1}$ for some $\theta \in(0, \pi)$ and $\xi^{\prime}=\left(\xi_{1}^{\prime}, \ldots, \xi_{q}^{\prime}\right) \in \mathbb{S}^{q-1}$ where $\left\{e_{1}, e_{2}, \ldots, e_{q+1}\right\}$ is the standard basis for $\mathbb{R}^{q+1}$. This observation drives us to consider the map $\varphi:(0, \pi) \times \mathbb{S}^{q-1} \rightarrow \mathbb{S}^{q}$ defined by

$$
\varphi\left(\theta, \xi^{\prime}\right)=\left(\cos \theta, \xi_{1}^{\prime} \sin \theta, \ldots, \xi_{q}^{\prime} \sin \theta\right)
$$

which induces the geodesic polar coordinate system on $\mathbb{S}^{q}$. This also provides a polar decomposition of the normalised measure $d \sigma_{q}$ on $\mathbb{S}^{q}$ as follows: Given a suitable function $f$ on $\mathbb{S}^{q}$ we have

$$
\int_{\mathbb{S} q} f(\xi) d \sigma_{q}(\xi)=\int_{0}^{\pi} \int_{\mathbb{S}^{q-1}} F\left(\theta, \xi^{\prime}\right)(\sin \theta)^{q-1} d \sigma_{q-1}\left(\xi^{\prime}\right) d \theta
$$

where $F=f \circ \varphi$. Also in this coordinate system, we have the following representation of the Laplace-Beltrami operator

$$
\Delta_{\mathbb{S} q}=\frac{\partial^{2}}{\partial \theta^{2}}+(q-1) \frac{\cos \theta}{\sin \theta} \frac{\partial}{\partial \theta}+\frac{1}{\sin ^{2} \theta} \Delta_{\mathbb{S}^{q-1}}
$$

With these notations we have the following analogue of Chernoff's theorem for spheres.

Theorem 1.9. Let $f \in C^{\infty}\left(\mathbb{S}^{q}\right)$ be such that $\Delta_{\mathbb{S}^{d}}^{m} f \in L^{2}\left(\mathbb{S}^{q}\right)$ for all $m \geq 0$ and satisfies the Carleman condition $\sum_{m=1}^{\infty}\left\|\Delta_{\mathbb{S}_{q}}^{m} f\right\|_{2}^{-\frac{1}{2 m}}=\infty$. If $\left.\frac{\partial^{m}}{\partial \theta^{m}}\right|_{\theta=0} F\left(\theta, \xi^{\prime}\right)=0$ for all $m \geq 0$ and for all $\xi^{\prime} \in \mathbb{S}^{q-1}$, then $f$ is identically zero. 
Observe that the vanishing condition imposed on $F$ simply means that when expressed in the geodesic polar coordinate system, all the derivatives of $f$ with respect to $\theta$ vanish on all great circles about the origin. Thus, we see that Theorem 1.9 serves as a good analogue of Chernoff theorem when the symmetric space $S$ is the sphere $\mathbb{S}^{q}$. However, in the general case of projective spaces we are not lucky enough to prove an exact analogue of Chernoff's theorem.

The geometry of the projective spaces is much more complicated than that of the sphere. It is almost impossible to find the exact vanishing condition as in the sphere case, for the projective spaces from the available information about those spaces from the point of view of Chernoff's theorem. As a matter of fact, the problem of proving an exact analogue of Chernoff's theorem for those spaces is still open and we plan to take up this problem in some future work. However, drawing analogy from the sphere case, for the other projective spaces, we use the corresponding product space $Y$ to formulate a theorem which is somewhat related to Chernoff's theorem. Although the theorem is not an exact counterpart of Chernoff's theorem for projective spaces, it illuminates the difficulties in obtaining an exact analogue for the theorem in consideration. This will be done in the appendix.

We complete this introduction with a brief description of the plan of the paper. In Section 2 we recall the requisite preliminaries on noncompact Riemannian symmetric spaces and in Section 3 we prove our version of Chernoff's theorem for the LaplaceBeltrami operator. In Section 4, after recalling necessary results from the theory of spherical harmonics and Jacobi polynomials and setting up the notations, we prove Theorem 1.9. We refer the reader to the papers [11] and [12] for related ideas.

\section{Preliminaries on Riemannian symmetric spaces of non-compact type}

In this section we describe the relevant theory regarding the harmonic analysis on rank one Riemannian symmetric spaces of noncompact type. General references for this section are the monographs of Helgason [18] and [19].

Let $G$ be a connected, noncompact semisimple Lie group with finite centre. Suppose $\mathfrak{g}$ denotes its Lie algebra. With respect to a fixed Cartan involution $\theta$ on $\mathfrak{g}$ we have the decomposition $\mathfrak{g}=\mathfrak{k} \oplus \mathfrak{p}$. Here $\mathfrak{k}$ and $\mathfrak{p}$ are the +1 and -1 eigenspaces of $\theta$ respectively. Let $\mathfrak{a}$ be the maximal abelian subspace of $\mathfrak{p}$. Also assume that the dimension of $\mathfrak{a}$ is one. Now we know that the involution $\theta$ induces an automorphism $\Theta$ on $G$ and $K=$ $\{g \in G: \Theta(g)=g\}$ is a maximal compact subgroup of $G$. We consider the homogeneous space $X=G / K$ which a is a smooth manifold endowed with a $G$-Riemannian metric induced by the restriction of the Killing form $\mathfrak{B}$ of $\mathfrak{g}$ on $\mathfrak{p}$. This turns $X$ into a rank one Riemannian symmetric space of noncompact type and every such space can be realised this way. 
Let $\mathfrak{a}^{*}$ denote the dual of $\mathfrak{a}$. Given $\alpha \in \mathfrak{a}^{*}$ we define

$$
\mathfrak{g}_{\alpha}:=\{X \in \mathfrak{g}:[Y, X]=\alpha(Y) X, \forall Y \in \mathfrak{a}\}
$$

Now $\Sigma:=\left\{\alpha \in \mathfrak{a}^{*}: \mathfrak{g}_{\alpha} \neq\{0\}\right\}$ is the set of all restricted roots of the pair $(\mathfrak{g}, \mathfrak{a})$. Let $\Sigma_{+}$ denote the set of all positive roots with respect to a fixed Weyl chamber. It is known that $\mathfrak{n}:=\oplus_{\alpha \in \Sigma_{+}} \mathfrak{g}_{\alpha}$ is a nilpotent subalgebra of $\mathfrak{g}$ and we have the Iwasawa decomposition $\mathfrak{g}=\mathfrak{k} \oplus \mathfrak{a} \oplus \mathfrak{n}$. Now writing $N=\exp \mathfrak{n}$ and $A=\exp \mathfrak{a}$ we obtain $G=K A N$ where $A$ is abelian and $N$ is a nilpotent subgroup of $G$. Moreover, $A$ normalizes $N$. In view of this decomposition every $g \in G$ can be uniquely written as $g=k(g) \exp H(g) n(g)$ where $H(g)$ belongs to $\mathfrak{a}$. Also we have $G=N A K$ and with respect to this decomposition we write $g \in N \exp A(g) K$ where the functions $A$ and $H$ are related via $A(g)=-H\left(g^{-1}\right)$. Now in the rank one case when dimension of $\mathfrak{a}$ is one, $\Sigma$ is given by either $\{ \pm \gamma\}$ or $\{ \pm \gamma, \pm 2 \gamma\}$ where $\gamma$ belongs to $\Sigma_{+}$. Let $\rho:=\left(m_{\gamma}+m_{2 \gamma}\right) / 2$ where $m_{\gamma}$ and $m_{2 \gamma}$ denote the multiplicities of the roots $\gamma$ and $2 \gamma$ respectively. The Haar measure $d g$ on $G$ is given by

$$
\int_{G} f(g) d g=\int_{K} \int_{A} \int_{N} f\left(k a_{t} n\right) e^{2 \rho t} d k d t d n .
$$

The measure $d x$ on $X$ is induced from the Haar measure $d g$ via the relation

$$
\int_{G} f(g K) d g=\int_{X} f(x) d x .
$$

Suppose $M$ denotes the centralizer of $A$ in $K$. The polar decomposition of $G$ reads as $G=K A K$ in view of which we can write each $g \in G$ as $g=k_{1} a_{r} k_{2}$ with $k_{1}, k_{2} \in K$. Actually the map $\left(k_{1}, a_{r}, k_{2}\right) \rightarrow k_{1} a_{r} k_{2}$ of $K \times A \times K$ into $G$ induces a diffeomorphism of $K / M \times A_{+} \times K$ onto an open dense subset of $G$ where $A_{+}=\exp \mathfrak{a}_{+}$and $\mathfrak{a}_{+}$is the fixed positive Weyl chamber which basically can be identified with $(0, \infty)$ in our case.

It is also well-known that each $\mathbf{X} \in \mathfrak{g}$ gives rise to a left invariant vector field on $G$ by the prescription

$$
\mathbf{X} f(g)=\left.\frac{d}{d t}\right|_{t=0} f(g \cdot \exp (t \mathbf{X})), g \in G .
$$

Since $\mathfrak{a}$ is one dimensional, we fix a basis $\{H\}$ of $\mathfrak{a}$. By an abuse of notation, we denote the left invariant vector field corresponding to this basis element by $H$. In fact, we can write $A=\left\{a_{r}=\exp (r H): r \in \mathbb{R}\right\}$.

\subsection{Helgason Fourier transform}

Define the function $A: X \times K / M \rightarrow \mathfrak{a}$ by $A(g K, k M)=A\left(k^{-1} g\right)$. Note that $A$ is right $K$-invariant in $g$ and right $M$-invariant in $K$. In what follows we denote the 
elements of $X$ and $K / M$ by $x$ and $b$ respectively. Let $\mathfrak{a}^{*}$ denote the dual of $\mathfrak{a}$ and $\mathfrak{a}_{\mathbb{C}}^{*}$ be its complexification. Here in our case $\mathfrak{a}^{*}$ and $\mathfrak{a}_{\mathbb{C}}^{*}$ can be identified with $\mathbb{R}$ and $\mathbb{C}$ respectively. For each $\lambda \in \mathfrak{a}_{\mathbb{C}}^{*}$ and $b \in K / M$, the function $x \rightarrow e^{(i \lambda+\rho) A(x, b)}$ is a joint eigenfunction of all invariant differential operators on $X$. For $f \in C_{c}^{\infty}(X)$, its Helgason Fourier transform is a function $\tilde{f}$ on $\mathfrak{a}_{\mathbb{C}}^{*} \times K / M$ defined by

$$
\tilde{f}(\lambda, b)=\int_{X} f(x) e^{(-i \lambda+\rho) A(x, b)} d x, \lambda \in \mathfrak{a}_{\mathbb{C}}^{*}, b \in K / M .
$$

Moreover, we know that if $f \in L^{1}(X)$ then $\widetilde{f}(., b)$ is a continuous function on $\mathfrak{a}^{*}$ which extends holomorphically to a domain containing $\mathfrak{a}^{*}$. The inversion formula for $f \in C_{c}^{\infty}(X)$ says that

$$
f(x)=c_{X} \int_{-\infty}^{\infty} \int_{K / M} \tilde{f}(\lambda, b) e^{(i \lambda+\rho) A(x, b)}|c(\lambda)|^{-2} d b d \lambda
$$

where $d \lambda$ stands for usual Lebesgue measure on $\mathbb{R}$ (i.e., $\mathfrak{a}^{*}$ ), $d b$ is the normalised measure on $K / M$ and $c(\lambda)$ is the Harish-Chandra $c$-function. The constant $c_{X}$ appearing in the above formula is explicit and depends on the symmetric space $X$ (See e.g., [19]). Also for $f \in L^{1}(X)$ with $\tilde{f} \in L^{1}\left(\mathfrak{a}^{*} \times K / M,|c(\lambda)|^{-2} d b d \lambda\right)$, the above inversion formula holds for a.e. $x \in X$. Furthermore, the mapping $f \rightarrow \widetilde{f}$ extends as an isometry of $L^{2}(X)$ onto $L^{2}\left(\mathfrak{a}_{+}^{*} \times K / M,|c(\lambda)|^{-2} d \lambda d b\right)$ which is known as the Plancherel theorem for the Helgason Fourier transform.

We also need to use certain irreducible representations of $K$ with $M$-fixed vectors. Suppose $\widehat{K_{0}}$ denotes the set of all irreducible unitary representations of $K$ with $M$ fixed vectors. Let $\delta \in \widehat{K_{0}}$ and $V_{\delta}$ be the finite dimensional vectors space on which $\delta$ is realised. As can be seen in [23, Theorem 6], the subspace of $V_{\delta}$ consisting of $M$-fixed vectors, has dimension one. Hence $V_{\delta}$ contains a unique normalised $M$-fixed vector $v_{1}$. Consider an orthonormal basis $\left\{v_{1}, v_{2}, \ldots, v_{d_{\delta}}\right\}$ for $V_{\delta}$. For $\delta \in \widehat{K_{0}}$ and $1 \leq j \leq d_{\delta}$, we define

$$
Y_{\delta, j}(k M)=\left(v_{j}, \delta(k) v_{1}\right), k M \in K / M
$$

It can be easily checked that $Y_{\delta, 1}(e K)=1$ and moreover, $Y_{\delta, 1}$ is $M$-invariant.

Proposition 2.1. The set $\left\{Y_{\delta, j}: 1 \leq j \leq d_{\delta}, \delta \in \widehat{K}_{0}\right\}$ forms an orthonormal basis for $L^{2}(K / M)$.

We refer the reader to [20, Theorem 3.5, Section 3, Chapter 5] for a proof of the aforementioned proposition. See also the discussion at the beginning of [19, Section 2, Chapter 3]. We can get an explicit realisation of $\widehat{K_{0}}$ by identifying $K / M$ with the unit sphere in $\mathfrak{p}$. By letting $\mathcal{H}^{m}$ to stand for the space of homogeneous harmonic polynomials of degree $m$ restricted to the unit sphere, we have the following spherical harmonic decomposition 


$$
L^{2}(K / M)=\oplus_{m=0}^{\infty} \mathcal{H}^{m}
$$

We know that each $V_{\delta}$ is contained in some $\mathcal{H}^{m}$ and hence the functions $Y_{\delta, j}$ can be identified with spherical harmonics.

Given $\delta \in \widehat{K_{0}}$ and $\lambda \in \mathfrak{a}_{\mathbb{C}}^{*}$ (i.e., $\mathbb{C}$ in our case) we consider the spherical functions of type $\delta$ defined by

$$
\Phi_{\lambda, \delta}(x):=\int_{K} e^{(i \lambda+\rho) A(x, k M)} Y_{\delta, 1}(k M) d k .
$$

These are eigenfunctions of the Laplace-Beltrami operator $\Delta_{X}$ with eigenvalue $-\left(\lambda^{2}+\rho^{2}\right)$. When $\delta$ is the trivial representation for which $Y_{\delta, 1}=1$, the function $\Phi_{\lambda, \delta}$ is called the elementary spherical function, denoted by $\Phi_{\lambda}$. More precisely,

$$
\Phi_{\lambda}(x)=\int_{K} e^{(i \lambda+\rho) A(x, k M)} d k
$$

Note that these functions are $K$-biinvariant. The spherical functions can be expressed in terms of Jacobi functions. In fact, if $x=g K$ and $g=k a_{r} k^{\prime}$ (polar decomposition), $\Phi_{\lambda, \delta}(x)=\Phi_{\lambda, \delta}\left(a_{r}\right)$. Suppose

$$
\alpha=\frac{1}{2}\left(m_{\gamma}+m_{2 \gamma}-1\right), \beta=\frac{1}{2}\left(m_{2 \gamma}-1\right) .
$$

For each $\delta \in \widehat{K_{0}}$ there exists a pair of integers $(p, q)$ such that

$$
\Phi_{\lambda, \delta}(x)=Q_{\delta}(i \lambda+\rho)(\alpha+1)_{p}^{-1}(\sinh r)^{p}(\cosh r)^{q} \varphi_{\lambda}^{(\alpha+p, \beta+q)}(r)
$$

where $\varphi_{\lambda}^{(\alpha+p, \beta+q)}$ are the Jacobi functions of type $(\alpha+p, \beta+q)$ and $Q_{\delta}$ are the Kostant polynomials given by

$$
Q_{\delta}(i \lambda+\rho)=\left(\frac{1}{2}(\alpha+\beta+1+i \lambda)\right)_{(p+q) / 2}\left(\frac{1}{2}(\alpha-\beta+1+i \lambda)\right)_{(p-q) / 2} .
$$

In the above we have used the notation $(z)_{m}=z(z+1)(z+2) \ldots(z+m-1)$. The following result proved in Helgason [19] will be very useful for our purpose:

Proposition 2.2. Let $\delta \in \widehat{K_{0}}$ and $1 \leq j \leq d_{\delta}$. Then we have

$$
\int_{K} e^{(i \lambda+\rho) A\left(x, k^{\prime} M\right)} Y_{\delta, j}\left(k^{\prime} M\right) d k^{\prime}=Y_{\delta, j}(k M) \Phi_{\lambda, \delta}\left(a_{r}\right), x=k a_{r} \in X .
$$

We refer the reader to the papers [16] and [17] for all the results recalled in this subsection. 


\subsection{Spherical Fourier transform}

We say that a function $f$ on $G$ is $K$-biinvariant if $f\left(k_{1} g k_{2}\right)=f(g)$ for all $k_{1}, k_{2} \in K$. It can be checked that if $f$ is a $K$-biinvariant integrable function then its Helgason Fourier transform $\tilde{f}(\lambda, b)$ is independent of $b \in K / M$ and by a little abuse of notation we write this as

$$
\tilde{f}(\lambda)=\int_{X} f(x) \Phi_{-\lambda}(x) d x .
$$

This is called the spherical Fourier transform. Now since $f$ is $K$ biinvariant, using the polar decomposition $g=k_{1} a_{r} k_{2}$, we can view $f$ as a function on $A$ alone: $f(g)=f\left(a_{r}\right)$. So the above integral takes the following polar form:

$$
\tilde{f}(\lambda)=\int_{0}^{\infty} f\left(a_{r}\right) \varphi_{\lambda}(r) w_{\alpha, \beta}(r) d r
$$

where $w_{\alpha, \beta}(r)=(2 \sinh r)^{2 \alpha+1}(2 \cosh r)^{2 \beta+1}$ and $\Phi_{-\lambda}\left(a_{r}\right)=\varphi_{\lambda}(r)$ are given by Jacobi function $\varphi_{\lambda}^{\alpha, \beta}(r)$ of type $(\alpha, \beta)$. Here $\alpha$ and $\beta$ are associated to the symmetric space as mentioned above. So it is clear that the spherical Fourier transform is basically Jacobi transform of type $(\alpha, \beta)$. In the rest of the section we describe certain results from the theory of Jacobi analysis.

Let $\alpha, \beta, \lambda \in \mathbb{C}$ and $-\alpha \notin \mathbb{N}$. The Jacobi functions $\varphi_{\lambda}^{(\alpha, \beta)}(r)$ of type $(\alpha, \beta)$ are solutions of the initial value problem

$$
\left(\mathcal{L}_{\alpha, \beta}+\lambda^{2}+\varrho^{2}\right) \varphi_{\lambda}^{(\alpha, \beta)}(r)=0, \varphi_{\lambda}^{(\alpha, \beta)}(0)=1
$$

where $\mathcal{L}_{\alpha, \beta}$ is the Jacobi operator defined by

$$
\mathcal{L}_{\alpha, \beta}:=\frac{d^{2}}{d r^{2}}+((2 \alpha+1) \operatorname{coth} r+(2 \beta+1) \tanh r) \frac{d}{d r}
$$

and $\varrho=\alpha+\beta+1$. Thus Jacobi functions $\varphi_{\lambda}^{(\alpha, \beta)}$ are eigenfunctions of $\mathcal{L}_{\alpha, \beta}$ with eigenvalues $-\left(\lambda^{2}+\varrho^{2}\right)$. These are even functions on $\mathbb{R}$ and are expressible in terms of hypergeometric functions. For certain values of the parameters $(\alpha, \beta)$ these functions arise naturally as spherical functions on Riemannian symmetric spaces of noncompact type. The Jacobi transform of a suitable function $f$ on $\mathbb{R}^{+}$is defined by

$$
J_{\alpha, \beta} f(\lambda)=\int_{0}^{\infty} f(r) \varphi_{\lambda}^{(\alpha, \beta)}(r) w_{\alpha, \beta}(r) d r
$$

This is also called the Fourier-Jacobi transform of type $(\alpha, \beta)$. It can be checked that the operator $\mathcal{L}_{\alpha, \beta}$ is selfadjoint on $L^{2}\left(\mathbb{R}^{+}, w_{\alpha, \beta}(r) d r\right)$ and that 


$$
\widetilde{\mathcal{L}_{\alpha, \beta} f}(\lambda)=-\left(\lambda^{2}+\varrho^{2}\right) \tilde{f}(\lambda) .
$$

Under certain assumptions on $\alpha$ and $\beta$ the inversion and Plancherel formula for this transform take a nice form as described below.

Theorem 2.3 ([22]). Let $\alpha, \beta \in \mathbb{R}, \alpha>-1$ and $|\beta| \leq \alpha+1$. Suppose $c_{\alpha, \beta}(\lambda)$ denotes the Harish-Chandra $c$-function defined by

$$
c_{\alpha, \beta}(\lambda)=\frac{2^{\varrho-i \lambda} \Gamma(\alpha+1) \Gamma(i \lambda)}{\Gamma\left(\frac{1}{2}(i \lambda+\varrho)\right) \Gamma\left(\frac{1}{2}(i \lambda+\alpha-\beta+1)\right)}
$$

(1) (Inversion) For $f \in C_{0}^{\infty}(\mathbb{R})$ which is even we have

$$
f(r)=\frac{1}{2 \pi} \int_{0}^{\infty} J_{\alpha, \beta} f(\lambda) \varphi_{\lambda}^{(\alpha, \beta)}(r)\left|c_{\alpha, \beta}(\lambda)\right|^{-2} d \lambda
$$

(2) (Plancherel) For $f, g \in C_{0}^{\infty}(\mathbb{R})$ which are even, the following holds

$$
\int_{0}^{\infty} f(r) \overline{g(r)} w_{\alpha, \beta}(r) d r=\int_{0}^{\infty} J_{\alpha, \beta} f(\lambda) \overline{J_{\alpha, \beta} g(\lambda)}\left|c_{\alpha, \beta}(\lambda)\right|^{-2} d \lambda .
$$

The mapping $f \longmapsto \tilde{f}$ extends as an isometry from $L^{2}\left(\mathbb{R}^{+}, w_{\alpha, \beta}(r) d r\right)$ onto $L^{2}\left(\mathbb{R}^{+},\left|c_{\alpha, \beta}(\lambda)\right|^{-2} d \lambda\right)$.

We will make use of this theorem in proving an analogue of Chernoff's theorem for the Laplace-Beltrami operator $\Delta_{X}$ in the next section.

\section{Chernoff's theorem on rank one symmetric spaces of noncompact type}

In this section we prove our main theorem i.e., an analogue of Chernoff's theorem for $\Delta_{X}$. The main idea of the proof is to reduce the result for $\Delta_{X}$ to a result for Jacobi operator. So, first we indicate a proof of Chernoff's theorem for Jacobi operator.

Theorem 3.1. Let $\alpha, \beta \in \mathbb{R}, \alpha>-1$ and $|\beta| \leq \alpha+1$. Suppose $f \in L^{2}\left(\mathbb{R}^{+}, w_{\alpha, \beta}(r) d r\right)$ is such that $\mathcal{L}_{\alpha, \beta}^{m} f \in L^{2}\left(\mathbb{R}^{+}, w_{\alpha, \beta}(r) d r\right)$ for all $m \in \mathbb{N}$ and satisfies the Carleman condition $\sum_{m=1}^{\infty}\left\|\mathcal{L}_{\alpha, \beta}^{m} f\right\|_{2}^{-1 /(2 m)}=\infty$. If $\mathcal{L}_{\alpha, \beta}^{m} f(0)=0$ for all $m \geq 0$ then $f$ is identically zero.

Proof. Given $f$ as in the statement of the theorem, we consider the Borel measure $\mu_{f}$ defined on $\mathbb{R}$ given by

$$
\mu_{f}(E)=\int_{E}\left|J_{\alpha, \beta} f(\lambda)\right|\left|c_{\alpha, \beta}(\lambda)\right|^{-2} d \lambda
$$


where $E$ is a Borel subset of $\mathbb{R}$. Since the Fourier-Jacobi transform $J_{\alpha, \beta} f(\lambda)$ is an even function on $\mathbb{R}$, it is not hard to see that $\mu_{f}$ is an even measure. Let $M(m)$ stand for the $m$ th order moment of the measure. Then we see that

$$
\begin{aligned}
M(2 m) & =\int_{-\infty}^{\infty} t^{2 m} d \mu_{f}(t)=\int_{-\infty}^{\infty} \lambda^{2 m}\left|J_{\alpha, \beta} f(\lambda)\right|\left|c_{\alpha, \beta}(\lambda)\right|^{-2} d \lambda \\
& \leq \int_{-\infty}^{\infty}\left(\lambda^{2}+\varrho^{2}\right)^{m}\left|J_{\alpha, \beta} f(\lambda)\right|\left|c_{\alpha, \beta}(\lambda)\right|^{-2} d \lambda .
\end{aligned}
$$

But by Cauchy-Schwarz inequality followed by an application of plancherel formula we have

$$
M(2 m) \leq C_{j}\left(\int_{0}^{\infty}\left(\lambda^{2}+\varrho^{2}\right)^{2(m+j)}\left|J_{\alpha, \beta} f(\lambda)\right|^{2}\left|c_{\alpha, \beta}(\lambda)\right|^{-2} d \lambda\right)^{\frac{1}{2}}=C_{j}\left\|\mathcal{L}_{\alpha, \beta}^{(m+j)} f\right\|_{2}
$$

where $C_{j}^{2}=\int_{0}^{\infty}\left(\lambda^{2}+\varrho^{2}\right)^{-2 j}\left|c_{\alpha, \beta}(\lambda)\right|^{-2} d \lambda$ which is finite for large enough $j$ since $\left|c_{\alpha, \beta}(\lambda)\right|^{-2}$ has polynomial growth in $\lambda$. Now as in proof of [12, Theorem 2.1] one easily checks that $\sum_{m=1}^{\infty} M(2 m)^{-1 / 2 m}=\infty$ together with [15, Theorem 2.3] proves that polynomials are dense in $L^{1}\left(\mathbb{R}, d \mu_{f}\right)$ and hence even polynomials are dense in $L_{e}^{1}\left(\mathbb{R}, d \mu_{f}\right)$. Since $f \in L^{2}\left(\mathbb{R}^{+}, w_{\alpha, \beta}(r) d r\right)$, by Plancherel $J_{\alpha, \beta} f \in L_{e}^{2}\left(\mathbb{R},\left|c_{\alpha, \beta}(\lambda)\right|^{-2} d \lambda\right)$ which can be used to show that $\overline{J_{\alpha, \beta} f} \in L_{e}^{1}\left(\mathbb{R}, d \mu_{f}\right)$. Hence for any $\varepsilon>0$, there exists an even polynomial $q$ such that

$$
\int_{0}^{\infty}\left|\overline{J_{\alpha, \beta} f(\lambda)}-q(\lambda)\right|\left|J_{\alpha, \beta} f(\lambda)\right| \tilde{w}(\lambda) d \lambda<\varepsilon
$$

But notice that $\left|J_{\alpha, \beta} f(\lambda)\right|^{2}=\left(\overline{J_{\alpha, \beta} f(\lambda)}-q(\lambda)\right) J_{\alpha, \beta} f(\lambda)+q(\lambda) J_{\alpha, \beta} f(\lambda)$. So from the Plancherel formula, we see that

$$
\|f\|_{2}^{2} \leq \int_{0}^{\infty}\left|\overline{J_{\alpha, \beta} f(\lambda)}-q(\lambda)\right|\left|J_{\alpha, \beta} f(\lambda)\right|\left|c_{\alpha, \beta}(\lambda)\right|^{-2} d \lambda+\int_{0}^{\infty} q(\lambda) J_{\alpha, \beta} f(\lambda)\left|c_{\alpha, \beta}(\lambda)\right|^{-2} d \lambda .
$$

But the vanishing condition $\mathcal{L}_{\alpha, \beta}^{m} f(0)=0$ (interpreted as $\lim _{r \rightarrow 0^{+}} \mathcal{L}_{\alpha, \beta}^{m} f(r)=0$ ) for all $m \geq 0$ yields

$$
\int_{0}^{\infty}\left(\lambda^{2}+\varrho^{2}\right)^{m} J_{\alpha, \beta} f(\lambda) \varphi_{\lambda}(r)\left|c_{\alpha, \beta}(\lambda)\right|^{-2} d \lambda=0
$$

Now since $q$ is an even polynomial, there exists a polynomial $p$ such that $p\left(\lambda^{2}+\varrho^{2}\right)=q(\lambda)$. Hence the above equation (3.2) along with the fact that $\varphi_{\lambda}(0)=1$ gives 


$$
\int_{0}^{\infty} q(\lambda) J_{\alpha, \beta} f(\lambda)\left|c_{\alpha, \beta}(\lambda)\right|^{-2} d \lambda=\lim _{r \rightarrow 0} \int_{0}^{\infty} p\left(\lambda^{2}+\varrho^{2}\right) J_{\alpha, \beta} f(\lambda) \varphi_{\lambda}(r)\left|c_{\alpha, \beta}(\lambda)\right|^{-2} d \lambda=0
$$

which together with (3.1) proves that $\|f\|_{2}^{2}<\varepsilon$ which is true for every $\varepsilon>0$, proving that $f=0$.

In [12] the above result was proved under the assumption that $f$ vanishes near 0 which was good enough for us to prove Ingham's theorem which was our main concern there. But a close examination of the proof reveals that the assumption is superfluous as can be seen in the above proof. In order to prove our main result, the following estimate on the ratio of Harish-Chandra $c$-functions is also needed.

Lemma 3.2. Let $\alpha, \beta$ be as in (2.1) and $(p, q)$ be the pair of integers associated to $\delta \in \widehat{K_{0}}$. Then for any $\lambda \geq 0$ we have

$$
\frac{\left|c_{\alpha, \beta}(\lambda)\right|^{2}}{\left|c_{\alpha+p, \beta+q}(\lambda)\right|^{2}}\left|Q_{\delta}(i \lambda+\rho)\right|^{-2} \leq C
$$

where $C$ is a constant independent of $\lambda$ depending only on the parameters $(\alpha, \beta)$ and $(p, q)$.

Proof. First note that from the definition (2.3) of Kostant polynomials we have

$$
\left|Q_{\delta}(i \lambda+\rho)\right|=\prod_{j=0}^{\frac{p+q}{2}}\left(\left(B_{1}+j\right)^{2}+\frac{1}{4} \lambda^{2}\right)^{\frac{1}{2}} \prod_{j=0}^{\frac{p-q}{2}}\left(\left(B_{2}+j\right)^{2}+\frac{1}{4} \lambda^{2}\right)^{\frac{1}{2}}
$$

where $B_{1}=\frac{1}{2}(\alpha+\beta+1)$ and $B_{2}=\frac{1}{2}(\alpha-\beta+1)$. From the above expression, it can be easily checked that $\left|Q_{\delta}(i \lambda+\rho)\right| /\left(2^{-1} \lambda\right)^{p} \rightarrow 1$ as $\lambda \rightarrow \infty$ so that

$$
\left|Q_{\delta}(i \lambda+\rho)\right| \sim 2^{-p} \lambda^{p}, \quad \lambda \rightarrow \infty
$$

Moreover, we also have

$$
\left|Q_{\delta}(i \lambda+\rho)\right| \geq \prod_{j=0}^{\frac{p+q}{2}}\left|B_{1}+j\right| \prod_{j=0}^{\frac{p-q}{2}}\left|B_{2}+j\right|=\text { constant }
$$

Now using [5, Lemma 2.4] we have

$$
\frac{\left|c_{\alpha, \beta}(\lambda)\right|^{2}}{\left|c_{\alpha+p, \beta+q}(\lambda)\right|^{2}} \sim \lambda^{2 p}, \quad \lambda \rightarrow \infty
$$

which together with (3.3) implies that 


$$
\frac{\left|c_{\alpha, \beta}(\lambda)\right|^{2}}{\left|c_{\alpha+p, \beta+q}(\lambda)\right|^{2}}\left|Q_{\delta}(i \lambda+\rho)\right|^{-2} \sim 1, \quad \lambda \rightarrow \infty .
$$

Also the ratio in (3.4) being a continuous function of $\lambda$ is bounded near the origin. Hence the result follows.

Proof of Theorem 1.7. Let $f$ be as in the statement of the Theorem 1.7. We complete the proof in the following steps.

Step 1: Using Proposition 2.1 we write

$$
\widetilde{f}(\lambda, k)=\sum_{\delta \in \widehat{K_{0}}} \sum_{j=1}^{d_{\delta}} F_{\delta, j}(\lambda) Y_{\delta, j}(k)
$$

where $F_{\delta, j}(\lambda)$ are the spherical harmonic coefficients of $\tilde{f}(\lambda, \cdot)$ defined by

$$
F_{\delta, j}(\lambda)=\int_{K / M} \tilde{f}(\lambda, k) Y_{\delta, j}(k) d k
$$

Fix $\delta \in \widehat{K_{0}}$ and $1 \leq j \leq d_{\delta}$. From the definition of the Helgason Fourier transform we have

$$
F_{\delta, j}(\lambda)=\int_{K / M} \int_{G / K} f(x) e^{(-i \lambda+\rho) A(x, k M)} Y_{\delta, j}(k M) d x d k
$$

Now using Fubini's theorem, in view of the Proposition 2.2 the integral on the right hand side of above is equal to

$$
\int_{G / K} f(x) Y_{\delta, j}(k M) \Phi_{\lambda, \delta}\left(a_{r}\right) d x
$$

The function $g_{\delta, j}(x)$ defined by

$$
g_{\delta, j}(x)=\int_{K} f\left(k^{\prime} x\right) Y_{\delta, j}\left(k^{\prime} M\right) d k^{\prime}, x \in X
$$

is clearly $K$-biinvariant, and hence by abuse of notation we write

$$
g_{\delta, j}(r)=\int_{K} f\left(k^{\prime} a_{r}\right) Y_{\delta, j}\left(k^{\prime} M\right) d k^{\prime}
$$

Now performing the integral in (3.6) using polar coordinates we obtain 


$$
F_{\delta, j}(\lambda)=\int_{0}^{\infty} g_{\delta, j}(r) \Phi_{\lambda, \delta}\left(a_{r}\right) w_{\alpha, \beta}(r) d r
$$

Now recall that for each $\delta \in \widehat{K_{0}}$ there exist a pair of integers $(p, q)$ such that

$$
\Phi_{\lambda, \delta}(x)=Q_{\delta}(i \lambda+\rho)(\alpha+1)_{p}^{-1}(\sinh r)^{p}(\cosh r)^{q} \varphi_{\lambda}^{(\alpha+p, \beta+q)}(r) .
$$

By defining

$$
f_{\delta, j}(r)=\frac{4^{-(p+q)}}{(\alpha+1)_{p}} g_{\delta, j}(r)(\sinh r)^{-p}(\cosh r)^{-q}
$$

and recalling the definition of Jacobi transforms we obtain

$$
F_{\delta, j}(\lambda)=Q_{\delta}(i \lambda+\rho) J_{\alpha+p, \beta+q}\left(f_{\delta, j}\right)(\lambda)
$$

Step 2: In this step we estimate the $L^{2}$ norm of powers of Jacobi operator applied to $f_{\delta, j}$ in terms of the $L^{2}$ norm of corresponding powers of $\Delta_{X}$ applied to $f$. Let $m \in \mathbb{N}$. Note that the Plancherel formula 2.3 for the Jacobi transform yields

$$
\begin{aligned}
& \left.\left\|\mathcal{L}_{\alpha+p, \beta+q}^{m}\left(f_{\delta, j}\right)\right\|_{L^{2}\left(\mathbb{R}^{+}, w_{\alpha+p, \beta+q}\right.}(r) d r\right) \\
& =\left(\int_{0}^{\infty}\left(\lambda^{2}+\rho_{\delta}^{2}\right)^{2 m}\left|J_{\alpha+p, \beta+q}\left(f_{\delta, j}\right)(\lambda)\right|^{2}\left|c_{\alpha+p, \beta+q}(\lambda)\right|^{-2} d \lambda\right)^{\frac{1}{2}}
\end{aligned}
$$

where $\rho_{\delta}=\alpha+\beta+p+q+1$. In view of (3.9) the above integral reduces to

$$
\left(\left.\int_{0}^{\infty}\left(\lambda^{2}+\rho_{\delta}^{2}\right)^{2 m}\left|F_{\delta, j}(\lambda)\right|^{2}\left|Q_{\delta}(i \lambda+\rho)\right|^{-2} c_{\alpha+p, \beta+q}(\lambda)\right|^{-2} d \lambda\right)^{\frac{1}{2}}
$$

which after recalling the definition of $F_{\delta, j}(\lambda)$ reads as

$$
\left(\left.\int_{0}^{\infty}\left(\lambda^{2}+\rho_{\delta}^{2}\right)^{2 m}\left|Q_{\delta}(i \lambda+\rho)\right|^{-2}|| \int_{K} \tilde{f}(\lambda, k) Y_{\delta, j}(k) d k\right|^{2}\left|c_{\alpha+p, \beta+q}(\lambda)\right|^{-2} d \lambda\right)^{\frac{1}{2}}
$$

By an application of Minkowski's integral inequality, the above integral is dominated by

$$
\int_{K}\left(\int_{0}^{\infty}\left(\lambda^{2}+\rho_{\delta}^{2}\right)^{2 m}\left|Q_{\delta}(i \lambda+\rho)\right|^{-2}|\widetilde{f}(\lambda, k)|^{2}\left|c_{\alpha+p, \beta+q}(\lambda)\right|^{-2} d \lambda\right)^{\frac{1}{2}}\left|Y_{\delta, j}(k)\right| d k .
$$


Now using Cauchy-Schwarz inequality along with the fact that $\left\|Y_{\delta, j}\right\|_{L^{2}(K / M)}=1$, we see that the above integral is bounded by

$$
\left(\int_{K / M} \int_{0}^{\infty}\left(\lambda^{2}+\rho_{\delta}^{2}\right)^{2 m}\left|Q_{\delta}(i \lambda+\rho)\right|^{-2}|\tilde{f}(\lambda, k)|^{2}\left|c_{\alpha+p, \beta+q}(\lambda)\right|^{-2} d \lambda d k\right)^{\frac{1}{2}}
$$

Since $\frac{\lambda^{2}+\rho_{\delta}^{2}}{\lambda^{2}+\rho^{2}}=1+\frac{\rho_{\delta}^{2}-\rho^{2}}{\lambda^{2}+\rho^{2}}$ is a decreasing function of $\lambda$ it follows that $\frac{\lambda^{2}+d^{2}}{\lambda^{2}+\rho^{2}} \leq C(\alpha, \beta)$ with $C(\alpha, \beta)=\frac{(\alpha+\beta+p+q+1)^{2}}{(\alpha+\beta+1)^{2}}$. This together with the Lemma 3.2 yields the following estimate for the integral under consideration: for some constant $C_{1}=C_{1}(\alpha, \beta)$

$$
C_{1}^{m}\left(\int_{K / M} \int_{0}^{\infty}\left(\lambda^{2}+\rho^{2}\right)^{2 m}|\tilde{f}(\lambda, k)|^{2}\left|c_{\alpha, \beta}(\lambda)\right|^{-2} d \lambda d k\right)^{\frac{1}{2}}
$$

Finally, from the series of inequalities above, we obtain

$$
\left.\left\|\mathcal{L}_{\alpha+p, \beta+q}^{m}\left(f_{\delta, j}\right)\right\|_{L^{2}\left(\mathbb{R}^{+}, w_{\alpha+p, \beta+q}\right.}(r) d r\right) \leq C_{1}^{m}\left\|\Delta_{X}^{m} f\right\|_{2}
$$

Hence from the hypothesis of the theorem it follows that

$$
\sum_{m=1}^{\infty}\left\|\mathcal{L}_{\alpha+p, \beta+q}^{m}\left(f_{\delta, j}\right)\right\|_{L^{2}\left(\mathbb{R}^{+}, w_{\alpha+p, \beta+q}(r) d r\right)}^{-\frac{1}{2 m}}=\infty
$$

Step 3: Finally in this step we prove that $\mathcal{L}_{\alpha+p, \beta+q}^{m}\left(f_{\delta, j}\right)(0)=0$ for all $m \geq 0$. First recall that

$$
f_{\delta, j}(r)=\frac{4^{-(p+q)}}{(\alpha+1)_{p}}(\sinh r)^{-p}(\cosh r)^{-q} \int_{K} f\left(k a_{r}\right) Y_{\delta, j}(k M) d k
$$

As $\sinh r$ has a zero at the origin and $\cosh 0=1$, if we can show that as a function of $r$, the integral $\int_{K} f\left(k a_{r}\right) Y_{\delta, j}(k M) d k$ has a zero of infinite order at the 0 , then we are done. Now note that for any $m \in \mathbb{N}$

$$
\frac{d^{m}}{d r^{m}} \int_{K} f\left(k a_{r}\right) Y_{\delta, j}(k M) d k=\int_{K} \frac{d^{m}}{d r^{m}} f\left(k a_{r}\right) Y_{\delta, j}(k M) d k
$$

But by definition of the vector fields on $G$, writing $a_{r}=\exp (r H)$ we have

$$
\left.\frac{d^{m}}{d r^{m}} f\left(k a_{r}\right)\right|_{r=0}=\left.\frac{d^{m}}{d r^{m}} f(k \cdot \exp (r H))\right|_{r=0}=H^{m} f(k) .
$$


Hence by the hypothesis on $f$ we obtain $\left.\frac{d^{m}}{d r^{m}} f\left(k a_{r}\right)\right|_{r=0}=0$ for all $m$. Finally, proving $\mathcal{L}_{\alpha+p, \beta+q}^{m}\left(f_{\delta, j}\right)(0)=0$ is a routine matter: repeated application of L'Hospital rule gives the desired result.

Therefore, $f_{\delta, j}$ satisfies all the hypothesis of the Theorem 3.1 which allows us to conclude that $f_{\delta, j}=0$ i.e., $F_{\delta, j}=0$. As this is true for every $\delta \in \widehat{K_{0}}$ and $1 \leq j \leq d_{\delta}$ we get $f=0$ completing the proof of Theorem 1.2.

\section{Chernoff's theorem on sphere}

Our aim in this section is to prove an analogue of Chernoff's theorem for the Laplace-Beltrami operator on the sphere which is a compact symmetric space of rank one. We start with a brief description of Jacobi polynomial expansions in the following subsection.

\subsection{Jacobi polynomial expansion}

Let $\alpha, \beta>-1$. The Jacobi polynomials $P_{n}^{\alpha, \beta}$ of degree $n \geq 0$ and type $(\alpha, \beta)$ are defined by

$$
(1-x)^{\alpha}(1+x)^{\beta} P_{n}^{\alpha, \beta}(x)=\frac{(-1)^{n}}{2^{n} n !} \frac{d^{n}}{d x^{n}}\left\{(1-x)^{n+\alpha}(1+x)^{n+\beta}\right\}, x \in(-1,1) .
$$

By making a change of variable $x=\cos \theta$, it is convenient to work with the Jacobi trigonometric polynomials

$$
\mathcal{P}_{n}^{(\alpha, \beta)}(\theta)=C(\alpha, \beta, n) P_{n}^{(\alpha, \beta)}(\cos \theta)
$$

where $C(\alpha, \beta, n)$ is the normalising constant, explicitly given by

$$
C(\alpha, \beta, n)^{2}=\frac{(2 n+\alpha+\beta+1) \Gamma(n+1) \Gamma(n+\alpha+\beta+1)}{\Gamma(n+\alpha+1) \Gamma(n+\beta+1)} .
$$

Also it is worth pointing out that these polynomials are closely related to Gegenbauer's polynomials by the following formula

$$
C_{k}^{\lambda}(t)=\frac{\Gamma\left(\lambda+\frac{1}{2}\right) \Gamma(k+2 \lambda)}{\Gamma(2 \lambda) \Gamma\left(k+\lambda+\frac{1}{2}\right)} P_{k}^{\left(\lambda-\frac{1}{2}, \lambda-\frac{1}{2}\right)}(t), \lambda>-\frac{1}{2}, t \in(-1,1) .
$$

These Jacobi trigonometric polynomials are the eigenfunctions of the Jacobi differential operator given by

$$
\mathbb{L}_{\alpha, \beta}=-\frac{d^{2}}{d \theta^{2}}-\frac{\alpha-\beta+(\alpha+\beta+1) \cos \theta}{\sin \theta} \frac{d}{d \theta}
$$

with eigenvalues $n(n+\alpha+\beta+1)$ i.e., 


$$
\mathbb{L}_{\alpha, \beta} \mathcal{P}_{n}^{(\alpha, \beta)}=n(n+\alpha+\beta+1) \mathcal{P}_{n}^{(\alpha, \beta)},
$$

and $\left\{\mathcal{P}_{n}^{(\alpha, \beta)}: n \geq 0\right\}$ forms an orthonormal basis for the weighted $L^{2}$ space $L^{2}\left(\tilde{w}_{\alpha, \beta}\right):=$ $L^{2}\left((0, \pi), \tilde{w}_{\alpha, \beta}(\theta) d \theta\right)$ where the weight is given by

$$
\tilde{w}_{\alpha, \beta}(\theta)=\left(\sin \frac{\theta}{2}\right)^{2 \alpha+1}\left(\cos \frac{\theta}{2}\right)^{2 \beta+1} .
$$

As a consequence we have the following Plancherel formula valid for $f \in L^{2}\left(\tilde{w}_{\alpha, \beta}\right)$

$$
\int_{0}^{\pi}|f(\theta)|^{2} \tilde{w}_{\alpha, \beta}(\theta) d \theta=\sum_{n=0}^{\infty}\left|\mathcal{J}_{\alpha, \beta} f(n)\right|^{2}
$$

where $\mathcal{J}_{\alpha, \beta} f(n)$ denotes the Fourier-Jacobi coefficients defined by

$$
\mathcal{J}_{\alpha, \beta} f(n)=\int_{0}^{\pi} f(\theta) \mathcal{P}_{n}^{(\alpha, \beta)}(\theta) \tilde{w}_{\alpha, \beta}(\theta) d \theta, \quad n \geq 0 .
$$

We have the following version of Chernoff's theorem using the iterates of the Jacobi operator.

Theorem 4.1. Let $\alpha, \beta>-1$. Suppose $f \in L^{2}\left(\tilde{w}_{\alpha, \beta}\right)$ is such that $\mathbb{L}_{\alpha, \beta}^{m} f \in L^{2}\left(\tilde{w}_{\alpha, \beta}\right)$ for all $m \in \mathbb{N}$ and satisfies the Carleman condition $\sum_{m=1}^{\infty}\left\|\mathbb{L}_{\alpha, \beta}^{m} f\right\|_{2}^{-1 /(2 m)}=\infty$. If $\mathbb{L}_{\alpha, \beta}^{m} f(0)=0$ for all $m \geq 0$ then $f$ is identically zero.

This is the analogue of Theorem 3.1 for Jacobi polynomial expansions which plays an important role in proving Theorem 1.9 for compact Riemannian symmetric spaces. This result has been essentially proved in [11, Theorem 2.4] where the vanishing condition assumed is much stronger. However, one can imitate the proof given in [11, Theorem 2.4] to get a stronger result with a weaker vanishing condition as in Theorem 4.1. Nevertheless, for the sake of convenience of the reader, we provide a proof here.

Proof of Theorem 4.1: We proceed as in the proof of Theorem 3.1. Let $f$ be as in the statement of the theorem. Consider the measure $\nu_{f}$ on $\mathbb{R}$ defined as follows: for any Borel function $\varphi$ on $\mathbb{R}$

$$
\int_{-\infty}^{\infty} \varphi(t) d \nu_{f}(t)=\frac{1}{2} \sum_{n=0}^{\infty}\left(\varphi\left(\sqrt{\lambda_{n}}\right)+\varphi\left(-\sqrt{\lambda_{n}}\right)\right)\left|\mathcal{J}_{\alpha, \beta}(n)\right|
$$

where $\lambda_{n}$ stands for the eigenvalue $n(n+\alpha+\beta+1)>0, \forall n \geq 0$. Clearly $\lambda_{0}=0$ and $\lambda_{n}>$ 0 , for $n \geq 1$. Now for any $m \geq 1$ we compute 


$$
\int_{-\infty}^{\infty} t^{2 m} d \nu_{f}(t)=\sum_{n=0}^{\infty} \lambda_{n}^{m}\left|\mathcal{J}_{\alpha, \beta}(n)\right|=\sum_{n=1}^{\infty} \lambda_{n}^{-j} \lambda_{n}^{m+j}\left|\mathcal{J}_{\alpha, \beta}(n)\right| .
$$

Now apply Cauchy-Schwarz inequality to obtain the estimate

$$
M(2 m) \leq \sqrt{C_{j}}\left(\sum_{n=1}^{\infty} \lambda_{n}^{2(m+j)}\left|\mathcal{J}_{\alpha, \beta}(n)\right|^{2}\right)^{\frac{1}{2}}=\sqrt{C_{j}}\left\|\mathbb{L}^{m+j} f\right\|_{2}
$$

where $C_{j}=\sum_{n=1}^{\infty} \lambda_{n}^{-2 j}$ which is clearly finite for large enough $j$. Here $M(m)$ denotes the $m$ th order moment of the measure $\nu_{f}$. Finally, proceeding as in [11, Theorem 2.4] one easily checks that the moments of the measure $\nu_{f}$ satisfy the Carleman condition i.e., $\sum_{m=1}^{\infty} M(2 m)^{-1 / 2 m}=\infty$. Therefore, using [15, Theorem 2.3] we infer that polynomials are dense in $L^{1}\left(\mathbb{R}, d \nu_{f}\right)$. Now we take the even function $\varphi$ defined on the support of $\nu_{f}$ by $\varphi\left(\sqrt{\lambda_{n}}\right)=\varphi\left(-\sqrt{\lambda_{n}}\right)=\overline{\mathcal{J}_{\alpha, \beta} f(n)}$. Now it is clear that

$$
\int_{-\infty}^{\infty}|\varphi(t)| d \nu_{f}(t)=\sum_{n=0}^{\infty}\left|\mathcal{J}_{\alpha, \beta} f(n)\right|^{2}<\infty
$$

proving that $\varphi \in L^{1}\left(\mathbb{R}, d \nu_{f}\right)$. Also by definition $\varphi$ is even. Since $\nu_{f}$ is an even measure, even polynomials are dense in $L_{e}^{1}\left(\mathbb{R}, d \nu_{f}\right)$. Hence for any $\epsilon>0$ we can choose an even polynomial $q$ such that $\|\varphi-q\|_{L^{1}\left(\mathbb{R}, d \nu_{f}\right)}<\epsilon$ i.e.,

$$
\sum_{n=0}^{\infty}\left|\overline{\mathcal{J}_{\alpha, \beta} f(n)}-q\left(\sqrt{\lambda_{n}}\right)\right|\left|\mathcal{J}_{\alpha, \beta} f(n)\right|<\epsilon
$$

Now the vanishing condition $\mathbb{L}_{\alpha, \beta}^{m} f(0)=0$ (interpreted as $\lim _{r \rightarrow 0^{+}} \mathbb{L}_{\alpha, \beta}^{m} f(r)=0$ ) for all $m \geq 0$ translates into

$$
\sum_{n=0}^{\infty} \lambda_{n}^{m} \mathcal{J}_{\alpha, \beta} f(n)=0, \forall m \geq 0
$$

But since $q$ is even, the above allows us to conclude that $\sum_{n=0}^{\infty} q\left(\sqrt{\lambda_{k}}\right) \mathcal{J}_{\alpha, \beta} f(n)=0$. Now we write

$$
\left|\mathcal{J}_{\alpha, \beta} f(n)\right|^{2}=\left(\overline{\mathcal{J}_{\alpha, \beta} f(n)}-q\left(\sqrt{\lambda_{n}}\right)\right) \mathcal{J}_{\alpha, \beta} f(n)+q\left(\sqrt{\lambda_{n}}\right) \mathcal{J}_{\alpha, \beta} f(n)
$$

and make use of the above observations, we conclude that $\|f\|_{2}^{2}=\sum_{n=0}^{\infty}\left|\mathcal{J}_{\alpha, \beta} f(n)\right|^{2}<\epsilon$. As this is true for every $\epsilon$ it follows that $f=0$ proving the theorem. 


\subsection{The unit sphere $\mathbb{S}^{q}$}

Let $q \geq 2$. The unit sphere in $\mathbb{R}^{q+1}$ is given by

$$
\mathbb{S}^{q}:=\left\{\xi \in \mathbb{R}^{q+1}: \xi_{1}^{2}+\cdots+\xi_{q+1}^{2}=1\right\}
$$

The spherical harmonic decomposition reads as

$$
L^{2}\left(\mathbb{S}^{q}\right)=\bigoplus_{n=0}^{\infty} \mathcal{H}_{n}\left(\mathbb{S}^{q}\right)
$$

where $\mathcal{H}_{n}\left(\mathbb{S}^{q}\right)$ denotes the set of spherical harmonics of degree $n$. Now, for our purposes it is more convenient to work with the geodesic polar coordinate system on $\mathbb{S}^{q}$. Note that given $\xi \in \mathbb{S}^{q}$, we can write $\xi=(\cos \theta) e_{1}+\xi_{1}^{\prime}(\sin \theta) e_{2}+\ldots+\xi_{q}^{\prime}(\sin \theta) e_{q+1}$ for some $\theta \in(0, \pi)$ and $\xi^{\prime}=\left(\xi_{1}^{\prime}, \ldots, \xi_{q}^{\prime}\right) \in \mathbb{S}^{q-1}$ where $\left\{e_{1}, e_{2}, \ldots, e_{q+1}\right\}$ is the standard basis for $\mathbb{R}^{q+1}$. This observation drives us to consider the map $\varphi:(0, \pi) \times \mathbb{S}^{q-1} \rightarrow \mathbb{S}^{q}$ defined by

$$
\varphi\left(\theta, \xi^{\prime}\right)=\left(\cos \theta, \xi_{1}^{\prime} \sin \theta, \ldots, \xi_{q}^{\prime} \sin \theta\right)
$$

which induces the geodesic polar coordinate system on $\mathbb{S}^{q}$. This also provides a polar decomposition of the normalised measure $d \sigma_{q}$ on $\mathbb{S}^{q}$ as follows: Given a suitable function $f$ on $\mathbb{S}^{q}$ we have

$$
\int_{\mathbb{S}^{q}} f(\xi) d \sigma_{q}(\xi)=\int_{0}^{\pi} \int_{\mathbb{S}^{q-1}} F\left(\theta, \xi^{\prime}\right)(\sin \theta)^{q-1} d \sigma_{q-1}\left(\xi^{\prime}\right) d \theta
$$

where $F=f \circ \varphi$. Also in this coordinate system, we have the following representation of the Laplace-Beltrami operator

$$
\Delta_{\mathbb{S} q}=\frac{\partial^{2}}{\partial \theta^{2}}+(q-1) \cot \theta \frac{\partial}{\partial \theta}+\left(\sin ^{2} \theta\right)^{-1} \Delta_{\mathbb{S} q-1}
$$

The following theorem gives a representation of the spherical harmonics in this polar coordinate system.

Theorem 4.2. [21, Theorem 2.4] For $n \geq 0$ we have the following orthogonal decomposition

$$
\mathcal{H}_{n}\left(\mathbb{S}^{q}\right)=\bigoplus_{l=0}^{n} \mathcal{H}_{n, l}\left(\mathbb{S}^{q}\right)
$$

where the subspaces $\mathcal{H}_{n, l}\left(\mathbb{S}^{q}\right)$ are irreducible and invariant under $S O(q)$. Moreover, functions in $\mathcal{H}_{n, l}\left(\mathbb{S}^{q}\right)$ can be represented as 


$$
S(\xi)=(\sin \theta)^{l} C_{n-l}^{q / 2-1 / 2+l}(\cos \theta) S_{l}^{\prime}\left(\xi^{\prime}\right)
$$

where $\xi=\varphi\left(\theta, \xi^{\prime}\right)$ and $S_{l}^{\prime} \in \mathcal{H}_{l}\left(\mathbb{S}^{q-1}\right)$.

In view of the above theorem we have the orthogonal decomposition

$$
L^{2}\left(\mathbb{S}^{q}\right)=\bigoplus_{n=0}^{\infty} \bigoplus_{l=0}^{n} \mathcal{H}_{n, l}\left(\mathbb{S}^{q}\right)
$$

Now we set

$$
S_{n, l, k}(\xi)=a_{n, l}(\sin \theta)^{l} C_{n-l}^{l+\frac{q-1}{2}}(\cos \theta) S_{k, l}^{\prime}\left(\xi^{\prime}\right)
$$

where $\left\{S_{l, k}^{\prime}: 1 \leq k \leq N(l)\right\}$ is an orthonormal basis for $\mathcal{H}_{l}\left(\mathbb{S}^{q-1}\right)$. Here $a_{n, l}$ is the normalising constant so that $\left\|S_{n, l, k}\right\|_{L^{2}(\mathbb{S} q)}=1$ and it is explicitly given by

$$
a_{n, l}=\frac{2^{-\left(l+\frac{q-1}{2}\right)} \Gamma(2 l+q-1) \Gamma\left(n+\frac{q}{2}\right)}{\Gamma\left(l+\frac{q}{2}\right) \Gamma(n+l+q-1)} C\left(l+\frac{q-2}{2}, l+\frac{q-2}{2}, n-l\right) .
$$

We are now ready to prove Theorem 1.9. We state this here as well for the convenience of the reader.

Theorem 4.3. Let $f \in C^{\infty}\left(\mathbb{S}^{q}\right)$ be such that $\Delta_{\mathbb{S}^{d}}^{m} f \in L^{2}\left(\mathbb{S}^{q}\right)$ for all $m \geq 0$ and satisfies

$$
\sum_{m=1}^{\infty}\left\|\Delta_{\mathbb{S}^{q}}^{m} f\right\|_{2}^{-\frac{1}{2 m}}=\infty
$$

If $\left.\frac{\partial^{m}}{\partial \theta^{m}}\right|_{\theta=0} F\left(\theta, \xi^{\prime}\right)=0$ for all $m \geq 0$ and for all $\xi^{\prime} \in \mathbb{S}^{q-1}$, then $f$ is identically zero.

Proof. Let $f$ be as in the statement of the theorem. For $n \geq 0$, let $P_{n} f$ denote the projection of $f$ onto the space $\mathcal{H}_{n}\left(\mathbb{S}^{q}\right)$. Then from the above observations we have

$$
P_{n} f=\sum_{l=0}^{n} \sum_{k=1}^{N(l)}\left(f, S_{n, l, k}\right)_{L^{2}} S_{n, l, k}
$$

Also since $f \in L^{2}\left(\mathbb{S}^{q}\right)$ we have

$$
f=\sum_{n=0}^{\infty} P_{n} f=\sum_{n=0}^{\infty} \sum_{l=0}^{n} \sum_{k=1}^{N(l)}\left(f, S_{n, l, k}\right)_{L^{2}\left(\mathbb{S}^{q}\right)} S_{n, l . k}
$$

By interchanging the summations, we observe that 


$$
\begin{aligned}
& f=\sum_{l=0}^{\infty} \sum_{n=l}^{\infty} \sum_{k=1}^{N(l)}\left(f, S_{n, l, k}\right)_{L^{2}\left(\mathbb{S}^{q}\right)} S_{n, l . k} \\
& =\sum_{l=0}^{\infty} \sum_{n=0}^{\infty} \sum_{k=1}^{N(l)}\left(f, S_{n+l, l, k}\right)_{L^{2}(\mathbb{S} q)} S_{n+l, l . k} .
\end{aligned}
$$

In view of this, to prove the theorem it is enough to prove that $\left(f, S_{n+l, l, k}\right)_{L^{2}\left(\mathbb{S}^{q}\right)}=0$ for all $n, l, k$. To start with, let us first fix $n, l$ and $k$. From the expansion (4.7) we observe that

$$
\left(P_{n} f, S_{n+l, l, k}\right)_{L^{2}(\mathbb{S} q)}=\left(f, S_{n+l, l, k}\right)_{L^{2}\left(\mathbb{S}^{q}\right)}
$$

Next we use the expression for $S_{n+l, l, k}$ to show that these coefficients are nothing but Jacobi coefficients of a suitable function. In order to do so, we write the integral on $\mathbb{S}^{q}$ in polar coordinates to obtain

$$
\left(f, S_{n+l, l, k}\right)_{L^{2}\left(\mathbb{S}^{q}\right)}=\int_{0}^{\pi} \int_{\mathbb{S}^{q-1}} F\left(\theta, \xi^{\prime}\right) a_{n+l, l}(\sin \theta)^{l+q-1} C_{n}^{l+\frac{q-1}{2}}(\cos \theta) S_{k, l}^{\prime}\left(\xi^{\prime}\right) d \sigma_{q-1}\left(\xi^{\prime}\right) d \theta
$$

where $F:=f \circ \varphi$. Now using (4.3), (4.4) and (4.6), a simple calculation yields

$$
a_{n+l, l} C_{n}^{l+\frac{q-1}{2}}(\cos \theta)=2^{-\left(l+\frac{q-1}{2}\right)} C\left(l+\frac{q}{2}-1, l+\frac{q}{2}-1, n\right) P_{n}^{\left(l+\frac{q}{2}-1, l+\frac{q}{2}-1\right)}(\cos \theta)(4
$$

which transforms the above equation into

$$
\left(f, S_{n+l, l, k}\right)_{L^{2}(\mathbb{S} q)}=2^{-\left(l+\frac{q-1}{2}\right)} \int_{0}^{\pi} F_{k, l}(\theta)(\sin \theta)^{l+q-1} \mathcal{P}_{n}^{\left(l+\frac{d}{2}-1, l+\frac{d}{2}-1\right)}(\theta) d \theta
$$

where we have defined

$$
F_{k, l}(\theta):=\int_{\mathbb{S}^{q-1}} F\left(\theta, \xi^{\prime}\right) S_{k, l}^{\prime}\left(\xi^{\prime}\right) d \sigma_{q-1}\left(\xi^{\prime}\right)
$$

Now letting $\alpha=l+\frac{q}{2}-1$ and writing $\sin \theta=2 \sin \frac{\theta}{2} \cos \frac{\theta}{2}$ we see that

$$
(\sin \theta)^{l+q-1}=2^{l+q-1}(\sin \theta)^{-l} w_{\alpha, \alpha}(\theta)
$$

which together with (4.10) yields

$$
\left(f, S_{n+l, l, k}\right)_{L^{2}(\mathbb{S} q)}=\mathcal{J}_{\alpha, \alpha}\left(g_{k, l}\right)(n)
$$

where $g_{k, l}(\theta):=2^{\frac{q-1}{2}}(\sin \theta)^{-l} F_{k, l}(\theta)$. 
In view of the Plancherel formula (4.5) and the relation (4.8) we have

$$
\begin{aligned}
\left\|\mathbb{L}_{\alpha, \alpha}^{m} g_{l, k}\right\|_{2}^{2} & =\sum_{n=0}^{\infty}(n(n+2 \alpha+1))^{2 m}\left|\mathcal{J}_{\alpha, \alpha}\left(g_{l, k}\right)(n)\right|^{2} \\
& =\sum_{n=0}^{\infty}(n(n+2 l+q-1))^{2 m}\left|\int_{\mathbb{S}^{d}} P_{n} f(\xi) S_{n+l, l, k}(x) d \sigma_{q}(\xi)\right|^{2}
\end{aligned}
$$

By Cauchy-Schwarz inequality we note that

$$
\left|\int_{\mathbb{S}^{d}} P_{n} f(\xi) S_{n+l, l, k}(\xi) d \sigma_{q}(\xi)\right|^{2} \leq\left\|P_{n} f\right\|_{L^{2}\left(\mathbb{S}^{q}\right)}^{2}
$$

Finally, using the fact that $(n+2 l+q-1) \leq(n+q-1)\left(1+\frac{2 l}{q-1}\right)$ from (4.12) we get the estimate

$$
\left\|\mathbb{L}_{\alpha, \alpha}^{m} g_{l, k}\right\|_{2}^{2} \leq\left(1+\frac{2 l}{q-1}\right)^{2 m} \sum_{n=0}^{\infty}(n(n+q-1))^{2 m}\left\|P_{n} f\right\|_{L^{2}\left(\mathbb{S}^{q}\right)}^{2} .
$$

Therefore, we have proved

$$
\left\|\mathbb{L}_{\alpha, \alpha}^{m} g_{l, k}\right\|_{2} \leq\left(1+\frac{2 l}{q-1}\right)^{m}\left\|\Delta_{\mathbb{S}^{q}}^{m} f\right\|_{L^{2}\left(\mathbb{S}^{q}\right)}
$$

which by the hypothesis on the function $f$, implies that

$$
\sum_{m=1}^{\infty}\left\|\mathbb{L}_{\alpha, \alpha}^{m} g_{l, k}\right\|_{2}^{-\frac{1}{2 m}}=\infty
$$

Since $g_{l, k}(\theta)$ is related to $F\left(\theta, \xi^{\prime}\right)$ via the integral

$$
g_{l, k}(\theta)=2^{\frac{q-1}{2}}(\sin \theta)^{-l} \int_{\mathbb{S} q-1} F\left(\theta, \xi^{\prime}\right) S_{k, l}^{\prime}\left(\xi^{\prime}\right) d \sigma_{q-1}\left(\xi^{\prime}\right)
$$

the hypothesis $\left.\frac{\partial^{m}}{\partial \theta^{m}}\right|_{\theta=0} F\left(\theta, \xi^{\prime}\right)=0$ for all $m \geq 0$ allows us to conclude that $\mathbb{L}_{\alpha, \alpha}^{m} g_{l, k}(0)=$ 0 for all $m \geq 0$. Hence $g_{l, k}$ satisfies the hypotheses of Theorem 4.1 and hence we conclude that $g_{l, k}=0$ and consequently $\left(f, S_{n+l, l, k}\right)_{L^{2}\left(\mathbb{S}^{q}\right)}=0$. As this is true for any $n, l, k$, we conclude that $f=0$ completing the proof of the theorem. 


\section{Acknowledgments}

The authors would like to thank the referee for the valuable comments and suggestions which have been used in improving the presentation of the article. The first author is supported by Int. Ph.D. scholarship from Indian Institute of Science. The second author is thankful to DST-INSPIRE [DST/INSPIRE/04/2019/001914] for research grants. The third author is supported by J.C. Bose Fellowship from the Department of Science and Technology, Govt. of India (Grant no.: DSTO/PAM/TV/2036).

\section{Appendix A. General rank one compact symmetric spaces}

Let $S$ be a compact Riemannian manifold equipped with a Riemannian metric $d_{S}$. We say that $S$ is a two point homogeneous space if for any $x_{j}, y_{j} \in S, j=1,2$ with $d_{S}\left(x_{1}, x_{2}\right)=d_{S}\left(y_{1}, y_{2}\right)$, there exists $g \in I(S)$, the group of isometries of $S$ such that $g . x_{1}=y_{1}$, and $g \cdot x_{2}=y_{2}$ where $g . x$ denotes the usual action of $I(S)$ on $S$. It is well known that compact rank one symmetric spaces are compact two point homogeneous spaces (see Helgason [20]). Also these two point homogeneous spaces are completely classified by H.-C. Wang [28]. So, following Wang any compact rank one symmetric space $S$ is one of the following:

(1) the sphere $\mathbb{S}^{q} \subset \mathbb{R}^{q+1}, q \geq 1$;

(2) the real projective space $P_{q}(\mathbb{R}), q \geq 2$;

(3) the complex projective space $P_{l}(\mathbb{C}), l \geq 2$;

(4) the quaternionic projective space $P_{l}(\mathbb{H}), l \geq 2$;

(5) the Cauchy projective plane $P_{2}(\mathbb{C} a y)$.

In the previous section, we have considered the case of sphere and proved an analogue of Chernoff's theorem for the associated Laplace-Beltrami operator. As remarked in the introduction, we do not have exact analogue of Chernoff's theorem for the other rank one compact symmetric spaces. However, drawing analogies from the sphere case some partial results can be obtained, which are not exactly analogue of Chernoff's theorem but somewhat related to Chernoff's theorem. In this appendix, we take up the opportunity to describe this result for the projective spaces.

\section{A.1. The real projective spaces $P_{q}(\mathbb{R})$}

Let $O(q)$ denote the group of $q \times q$ orthogonal matrices. Then $P_{q}(\mathbb{R})$ can be identified with $S O(q+1) / O(q)$ which makes this a compact symmetric space. Now it is wellknown that the real projective space $P_{q}(\mathbb{R})$ can be obtained from $\mathbb{S}^{q}$ by identifying the antipodal points i.e., $P_{q}(\mathbb{R})=\mathbb{S}^{q} /\{ \pm I\}$ and the projection map $s \rightarrow \pm s$ from $\mathbb{S}^{q}$ to $P_{q}(\mathbb{R})$ is locally an isometry. So, the functions on $P_{q}(\mathbb{R})$ can be viewed as even functions on the corresponding sphere $\mathbb{S}^{q}$ and if $f_{e}$ is the even function on $\mathbb{S}^{q}$ corresponding to the 
function $f$ on $P_{q}(\mathbb{R})$ then $\Delta_{P_{q}(\mathbb{R})} f=\Delta_{\mathbb{S}^{q}} f_{e}$. Hence the analogue of Chernoff's theorem on $P_{q}(\mathbb{R})$ follows directly from the case of sphere.

\section{A.2. The other projective spaces $P_{l}(\mathbb{C}), P_{l}(\mathbb{H})$, and $P_{2}(\mathbb{C} a y)$}

This subsection is dedicated to prove an analogue of Theorem 1.9. In what follows, we first describe the connection between the projectives spaces and the associated polar form. As pointed out by T. O. Sherman in [27], analysis on these three projective spaces is quite similar. Closely following the notations of [27] (see also [9]), we first describe the appropriate polar coordinate representation of these spaces and then as in the sphere case we prove the Chernoff's theorem for the associated Laplace-Beltrami operators. To begin with, let $S$ denote any of these three spaces $P_{l}(\mathbb{C}), P_{l}(\mathbb{H})$, and $P_{2}(\mathbb{C} a y)$. Suppose $\Delta_{S}$ denotes the corresponding Laplace-Beltrami operator. Let $d \mu$ denote the normalised Riemann measure on $S$. We have the following orthogonal decomposition:

$$
L^{2}(S, d \mu):=L^{2}(S)=\bigoplus_{n=0}^{\infty} \mathcal{H}_{n}(S)
$$

where $\mathcal{H}_{n}(S)$ are finite dimensional and eigenspaces of $\Delta_{S}$ with eigenvalue $-n(n+k+q)$ where $q=2,4,8, k=l-2,2 l-3,3$, for $P_{l}(\mathbb{C}), P_{l}(\mathbb{H})$ and $P_{2}(\mathbb{C} a y)$, respectively. So, $\mathcal{H}_{n}(S)$ are the eigenspaces of $\Delta_{S}$ with eigenvalue $-n(n+k+q)$.

Let $\Omega:=\left\{x \in \mathbb{R}^{q+1}:|x| \leq 1\right\}$ be the closed unit ball in $\mathbb{R}^{q+1}$. We consider a weight function $w$ defined by $w(r):=r^{-1}(1-r)^{k}$ for $0<r \leq 1$. With these notations we have the following result proved in [27, Lemma 4.15].

Proposition A.1. There is a bounded linear map $E: L^{1}(S) \rightarrow L^{1}(\Omega, w(|x|) d x)$ satisfying

(1) For $f \in L^{1}(S)$,

$$
\int_{S} f d \mu=\int_{\Omega} E(f)(x) w(|x|) d x
$$

(2) The norm of $E$ as a map from $L^{p}(S)$ to $L^{p}(\Omega, w(|x|) d x)$ is $1(1 \leq p \leq \infty)$.

The integration formula in the above proposition is very useful. In fact, integrating the right hand side of that formula in polar coordinates we have

$$
\int_{\Omega} E(f)(x) w(|x|) d x=\int_{0}^{1} \int_{\mathbb{S}^{q}} E(f)(r \xi) w(r) r^{q} d \sigma_{q}(\xi) d r .
$$

Now a change of variables $r=\sin ^{2}(\theta / 2)$ allows us to conclude that 


$$
\int_{S} f d \mu=\int_{0}^{\pi} \int_{S^{q}} F(\theta, \xi)\left(\sin \frac{\theta}{2}\right)^{2 q-1}\left(\cos \frac{\theta}{2}\right)^{2 k+1} d \theta d \sigma_{q}(\xi),
$$

where $F(\theta, \xi)=E(f)\left(\sin ^{2}(\theta / 2) \xi\right)$. In [27], Sherman has described the image of $\mathcal{H}_{n}(S)$ under the map $E$. It has been proved that $E\left(\mathcal{H}_{n}(S)\right)=\mathcal{H}_{n}(\Omega, w)$ where $\mathcal{H}_{n}(\Omega, w)$ is the orthocomplement of $\mathbb{P}_{n-1}(\Omega)$ in $\mathbb{P}_{n}(\Omega)$ with respect to the inner product in $L^{2}(\Omega, w(|x|) d x)$. Here $\mathbb{P}_{n}(\Omega)$ denotes the set of all polynomials on $\Omega$ of degree up to $n$. Also note that in these trigonometric polar coordinates we can identify $\Omega$ with $Y:=(0, \pi) \times \mathbb{S}^{q}$ and

$$
d \omega(\theta, \xi):=\left(\sin \frac{\theta}{2}\right)^{2 q-1}\left(\cos \frac{\theta}{2}\right)^{2 k+1} d \theta d \sigma_{q}(\xi)
$$

is the corresponding measure on $Y$. Basically in view of this trigonometric polar coordinates we have $\mathcal{H}_{n}(\Omega, w)=\mathcal{H}_{n}(Y, \omega)$. These spaces are eigenspaces of the following differential operator

$$
\Lambda_{S}=\frac{\partial^{2}}{\partial \theta^{2}}+\frac{(q-1-k)+(q+k) \cos \theta}{\sin \theta} \frac{\partial}{\partial \theta}+\frac{1}{\sin ^{2}(\theta / 2)} \Delta_{\mathbb{S} q}
$$

with eigenvalues $n(n+k+q)$. The relation between this operator and the LaplaceBeltrami operator is described in the following proposition.

Proposition A.2. Let $f \in C^{2}(S)$ and $E$ be as in the Proposition A.1. Then we have

$$
E\left(\Delta_{S} f\right)=\Lambda_{S} E(f)
$$

For a proof of this fact we refer the reader to [27, Lemma 4.25]. Thus we have the following orthogonal decomposition

$$
L^{2}(Y, d \omega)=\bigoplus_{n=0}^{\infty} \mathcal{H}_{n}(Y, \omega)
$$

Moreover, $\mathcal{H}_{n}(Y, \omega)$ admits a further decomposition as $\mathcal{H}_{n}(Y, \omega)=\bigoplus_{j=0}^{n} \mathcal{H}_{n, j}(Y, \omega)$ where each $\mathcal{H}_{n, j}(Y, \omega)$ is irreducible under $S O(q+1)$ and spanned by $\left\{Q_{n, j, l}: 1 \leq l \leq\right.$ $N(j)\}$ (see [27, Theorem 4.22]) where for $x=\sin ^{2}(\theta / 2) \xi, \theta \in(0, \pi)$ and $\xi \in \mathbb{S}^{q}$

$$
\begin{aligned}
Q_{n, j, l}(x) & =b_{n, j}\left(\sin \frac{\theta}{2}\right)^{2 j} P_{n-j}^{(k, q-1+2 j)}\left(2 \sin ^{2}(\theta / 2)-1\right) S_{j, l}(\xi) \\
& =(-1)^{n-j} b_{n, j}\left(\sin \frac{\theta}{2}\right)^{2 j} P_{n-j}^{(q-1+2 j, k)}(\cos \theta) S_{j, l}(\xi)
\end{aligned}
$$


In the second equality we have used the symmetry relation for Jacobi polynomials i.e., $P_{n}^{(\alpha, \beta)}(-x)=(-1)^{n} P_{n}^{(\beta, \alpha)}(x)$. Here $\left\{S_{j, l}: 1 \leq l \leq N(j)\right\}$ a basis for $\mathcal{H}_{j}\left(\mathbb{S}^{q}\right)$, the spherical harmonics of degree $l$ on $\mathbb{S}^{q}$. The constants $b_{n, j}$ appearing in the above expression are chosen so that $\left\|Q_{n, j, l}\right\|_{2}=1$. In fact, it can be checked that $b_{n, j}=C(q-1+2 j, k, n-j)$. So, clearly $\left\{Q_{n, j, l}: n, l \geq 0,1 \leq N(l)\right\}$ forms an orthonormal basis for $L^{2}(Y, d \omega)$. Having equipped with the required machinery described above, we now proceed to prove the following result.

Theorem A.3. Let $Y=(0, \pi) \times \mathbb{S}^{q}$ and $\Lambda_{S}$ be as above. Suppose $f \in C^{\infty}(Y)$ satisfies $\Lambda_{S}^{m} f \in L^{2}(Y)$ for all $m \geq 0$ and $\sum_{m=1}^{\infty}\left\|\Lambda_{S}^{m} f\right\|_{2}^{-\frac{1}{2 m}}=\infty$. If the function $F(\theta, \xi)=$ $f\left(\left(\sin ^{2} \theta / 2\right) \xi\right),(\theta, \xi) \in Y$ associated to $f$ satisfies $\left.\frac{\partial^{m}}{\partial \theta^{m}}\right|_{\theta=0} F(\theta, \xi)=0$ for all $\xi \in \mathbb{S}^{q}$ and $m \geq 0$, then $f$ is identically zero.

Proof. Given a function $f$ with the property as in the statement of the theorem, we write $f\left(\sin ^{2}(\theta / 2) \xi\right)=F(\theta, \xi),(\theta, \xi) \in Y$. So, the analysis, described above allows us to write the projection of $F$ onto $\mathcal{H}_{n}(Y, \omega)$ as

$$
P_{n}^{S} F=\sum_{j=0}^{n} \sum_{l=1}^{N(j)}\left(F, Q_{n, j, l}\right) Q_{n, j, l}
$$

Now as in the sphere case, it is not hard to check that

$$
F=\sum_{j=0}^{\infty} \sum_{n=0}^{\infty} \sum_{l=1}^{N(j)}\left(F, Q_{n+j, j, l}\right)_{L^{2}(Y, d \omega)} Q_{n+j, j, l}
$$

Clearly, for each $n \geq 0$ we have

$$
\left(P_{n}^{S} F, Q_{n+j, j, l}\right)_{L^{2}(Y, d \omega)}=\left(F, Q_{n+j, j, l}\right)_{L^{2}(Y, d \omega)}
$$

As in the case of sphere, we will show that the right hand side of the above equation can be expressed as Jacobi coefficient of a suitable function related to $F$. By definition, we have

$$
\left(F, Q_{n+j, j, l}\right)=\int_{0}^{\pi} \int_{S^{q}} F(\theta, \xi) Q_{n+j, j, l}\left(\left(\sin ^{2} \frac{\theta}{2}\right) \xi\right)\left(\sin \frac{\theta}{2}\right)^{2 q-1}\left(\cos \frac{\theta}{2}\right)^{2 k+1} d \theta d \sigma_{q}(\xi)
$$

Now using the expression for $Q_{n+j, j, l}$ we have 


$$
\begin{aligned}
& \left(F, Q_{n+j, j, l}\right) \\
& \quad=(-1)^{j} b_{n+j, j} \int_{0}^{\pi} F_{j, l}(\theta)\left(\sin \frac{\theta}{2}\right)^{2 j} P_{n}^{(q-1+2 j, k)}(\cos \theta)\left(\sin \frac{\theta}{2}\right)^{2 q-1}\left(\cos \frac{\theta}{2}\right)^{2 k+1} d \theta .
\end{aligned}
$$

where $F_{j, l}$ are defined by

$$
F_{j, l}(\theta):=\int_{S^{q}} F(\theta, \xi) S_{j, l}(\xi) d \sigma_{q}(\xi)
$$

Writing $g_{j, l}(\theta)=(-1)^{j} F_{j, l}(\theta)\left(\sin \frac{\theta}{2}\right)^{-2 j}$ and using the definition of Jacobi coefficients we have

$$
\left(F, Q_{n+j, j, l}\right)_{L^{2}(Y, d \omega)}=\mathcal{J}_{\alpha, \beta}\left(g_{j, l}\right)(n)
$$

where $\alpha=q-1+2 j$ and $\beta=k$. Now using the Plancherel formula (4.5) along with (A.3) we obtain

$$
\begin{aligned}
\left\|\mathbb{L}_{\alpha, \beta}^{m} g_{j, l}\right\|_{2}^{2} & \left.=\sum_{n=0}^{\infty}(n(n+\alpha+\beta+1))\right)^{2 m}\left|\mathcal{J}_{\alpha, \beta}\left(g_{j, l}\right)(n)\right|^{2} \\
& =\sum_{n=0}^{\infty}(n(n+q+2 j+k))^{2 m}\left|\left(P_{n}^{S} F, Q_{n+j, j, l}\right)\right|^{2} .
\end{aligned}
$$

But $\left|\left(P_{n}^{S} f, Q_{n+j, j, l}\right)\right| \leq\left\|P_{n}^{S} f\right\|_{L^{2}(Y, d \omega)}$ and $(n+q+2 j+k) \leq\left(1+\frac{2 j}{q+k}\right)(n+q+k)$ so that we have

$$
\left\|\mathbb{L}_{\alpha, \beta}^{m} g_{j, l}\right\|_{2}^{2} \leq C^{2 m} \sum_{n=0}^{\infty}(n(n+q+k))^{2 m}\left\|P_{n}^{S} f\right\|_{2}^{2}=C^{2 m}\left\|\Lambda_{S}^{m} f\right\|_{2}^{2}
$$

where $C=\left(1+\frac{2 j}{q+k}\right)$ is a constant independent of $n$. Finally, the above equation along with the hypothesis allows us to conclude that

$$
\sum_{m=1}^{\infty}\left\|\mathbb{L}_{\alpha, \beta}^{m} g_{j, l}\right\|_{2}^{-\frac{1}{2 m}}=\infty
$$

Also using the hypothesis $\left.\frac{\partial^{m}}{\partial \theta^{m}}\right|_{\theta=0} F(\theta, \xi)=0$ for all $m \geq 0, \xi \in \mathbb{S}^{q}$, a simple calculation shows that $\mathbb{L}_{\alpha, \beta}^{m} g_{j, l}(0)=0$ for all $m \geq 0$. Hence by Theorem 4 .1, we have $g_{j, l}=0$ whence $\left(F, Q_{n+j, j, l}\right)_{L^{2}\left(\Omega_{0}, d \omega\right)}=0$. As this is true for all $n, j, l$ we conclude $f=0$. This completes the proof of the theorem.

Remark A.4. Ideally we would like to have a Chernoff's theorem for $S$ where the Carleman condition expressed in terms of $\Delta_{S}^{m}$ and the vanishing condition in terms of certain 
derivatives of $f$. As the operator $E: L^{2}(S) \rightarrow L^{2}(Y, d \omega)$ is bounded, in view of the relation $E\left(\Delta_{S}^{m} f\right)=\Lambda_{S}^{m} E(f)$, the Carleman condition in the theorem can be replaced by the condition $\sum_{m=1}^{\infty}\left\|\Delta_{S}^{m} f\right\|_{2}^{-\frac{1}{2 m}}=\infty$. However, as the operator $E$ is not known explicitly, the vanishing of the theta derivatives of $E(f)\left(\left(\sin ^{2} \theta / 2\right) \xi\right)$ cannot be expressed in terms of vanishing of $f$ and its derivatives. An examination of the construction of $E$ in [27] has convinced us that it is almost impossible to relate the derivatives of $f$ with those of $E(f)$.

\section{References}

[1] S. Bagchi, P. Ganguly, J. Sarkar, S. Thangavelu, On theorems of Chernoff and Ingham on the Heisenberg group, arXiv:2009.14230, 2020.

[2] M. Bhowmik, S. Pusti, S.K. Ray, Theorems of Ingham and Chernoff on Riemannian symmetric spaces of noncompact type, J. Funct. Anal. 279 (11) (2020).

[3] M. Bhowmik, S. Pusti, S.K. Ray, A theorem of Chernoff for quasi-analytic functions for Riemannian symmetric spaces, arXiv:2103.07667, 2021.

[4] S. Bochner, A.E. Taylor, Some theorems on quasi-analyticity for functions of several variables, Am. J. Math. 61 (2) (1939) 303-329.

[5] William O. Bray, Mark A. Pinsky, Pointwise Fourier Inversion on Rank One Symmetric Spaces and Related Topics, J. Funct. Anal. 151 (2) (1997) 306-333.

[6] T. Carleman, Les fonctions quasi-analytiques, volume 7 of Collection de monographies sur la theorie des fonctions publiée sous la direction de M.E. Borel Gauthier-Villars, Paris, 1926.

[7] P.R. Chernoff, Some remarks on quasi analytic functions, Trans. Am. Math. Soc. 167 (1972) 105-113.

[8] P.R. Chernoff, Quasi-analytic vectors and quasi-analytic functions, Bull. Am. Math. Soc. 81 (1975) 637-646.

[9] Ó. Ciaurri, L. Roncal, P.R. Stinga, Fractional integrals on compact Riemannian symmetric spaces of rank one, Adv. Math. 235 (2013) 627-647.

[10] A. Denjoy, Sur les fonctions quasi-analytiques de variable reelle, C.R. Acad. Sci. Paris 173 (1921) 1329-1331.

[11] P. Ganguly, S. Thangavelu, Theorems of Chernoff and Ingham for certain eigenfunction expansions, Adv. Math. 386 (2021), https://doi.org/10.1016/j.aim.2021.107815.

[12] P. Ganguly, S. Thangavelu, An uncertainty principle for spectral projections on rank one symmetric spaces of noncompact type, Ann. Mat. Pura Appl. (2021), https://doi.org/10.1007/s10231-02101116-3.

[13] P. Ganguly, S. Thangavelu, Analogues of theorems of Chernoff and Ingham on the Heisenberg group, accepted for publication in J. Anal. Math., arXiv:2106.02704.

[14] A.E. Ingham, A Note on Fourier Transforms, J. Lond. Math. Soc. S1-9 (1) (1934) 29-32, MR1574706.

[15] M. de Jeu, Determinate multidimensional measures, the extended Carleman theorem and quasianalytic weights, Ann. Probab. 31 (3) (2003) 1205-1227, MR1988469 (2004f:44006).

[16] K.D. Johnson, Composition series and intertwining operators for the spherical principal series II, Trans. Am. Math. Soc. 215 (1976) 269-283, MR0385012.

[17] K.D. Johnson, N. Wallach, Composition series and intertwining operators for the spherical principal series I, Trans. Am. Math. Soc. 229 (1977) 137-173, MR0447483.

[18] S. Helgason, Groups and Geometric Analysis, Academic Press, 1984, MR0754767.

[19] S. Helgason, Geometric analysis on Symmetric spaces, Math. Surveys Monographs, vol. 39, Amer. Math. Soc., 1994.

[20] S. Helgason, Differential Geometry, Lie Groups, and Symmetric Spaces, American Mathematical Soc., 2001.

[21] T.H. Koornwinder, The addition formula for Jacobi polynomials and spherical harmonics, SIAM J. Appl. Math. 25 (1973).

[22] T.H. Koornwinder, Jacobi functions and analysis on noncompact semisimple Lie groups, in: R. Askey, T.H. Koornwinder, W. Schempp (Eds.), Special Functions: Group Theoretical Aspects and Applications, Reidel, Dordrecht, 1984, pp. 1-85, MR0774055. 
[23] B. Kostant, On the existence and irreducibility of certain series of representations, Bull. Am. Math. Soc. 75 (1969) 627-642, MR0245725.

[24] D. Masson, W. Mc Clary, Classes of $C^{\infty}$ vectors and essential self-adjointness, J. Funct. Anal. 10 (1972) 19-32.

[25] A.E. Nussbaum, Quasi-analytic vectors, Ark. Mat. 6 (1965) 179-191, MR 33 No. 3105.

[26] W. Rudin, Real and Complex Analysis, 3rd edition, McGraw-Hill, Inc., 1987.

[27] T.O. Sherman, The Helgason Fourier transform for compact Riemannian symmetric spaces of rank one, Acta Math. 164 (1990).

[28] H.-C. Wang, Two-point homogeneous spaces, Ann. Math. (2) 55 (1952) 177-191. 